\title{
Structure and Physical Properties of Glasses in the System $\mathrm{Ag}_{2} \mathrm{Se}_{-}-\mathrm{Ga}_{2} \mathrm{Se}_{3}-\mathrm{GeSe}_{2}$
}

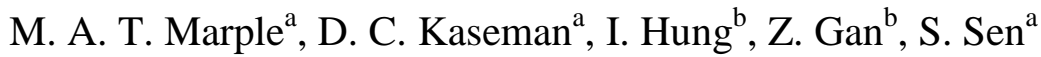 \\ ${ }^{a}$ Dept. of Materials Science \& Engineering, University of California at Davis, \\ Davis, CA 95616, USA \\ ${ }^{\mathrm{b}}$ Center for Interdisciplinary Magnetic Resonance, National High Magnetic Field Laboratory, \\ 1800 East Paul Dirac Drive, Tallahassee, FL 32310, USA
}




\begin{abstract}
Homogeneous glasses in the ternary system $\mathrm{Ag}_{2} \mathrm{Se}_{-} \mathrm{Ga}_{2} \mathrm{Se}_{3}-\mathrm{GeSe}_{2}$ are synthesized and their structure is characterized using Raman and one- and two-dimensional ${ }^{77} \mathrm{Se},{ }^{71} \mathrm{Ga}$ and ${ }^{69} \mathrm{Ga}$ nuclear magnetic resonance (NMR) spectroscopy. The structure of these glasses consists predominantly of a charge-compensated network of corner-sharing $(\mathrm{Ga} / \mathrm{Ge}) \mathrm{Se}_{4 / 2}$ tetrahedra where the negatively charged $\mathrm{GaSe}_{4 / 2}$ tetrahedra are charge balanced by the Ag cations. The deficiency in Se required to satisfy the tetrahedral coordination of $\mathrm{Ga}$ in glasses with $\mathrm{Ag}_{2} \mathrm{Se}: \mathrm{Ga}_{2} \mathrm{Se}_{3}<1$ is accommodated by the formation of homopolar Ge-Ge bonds. Progressive addition of $\mathrm{Ag}_{2} \mathrm{Se}$ to these glasses efficiently removes $\mathrm{Ge}-\mathrm{Ge}$ bonding such that their concentration essentially goes to zero at the chemical threshold $\mathrm{Ag}_{2} \mathrm{Se}: \mathrm{Ga}_{2} \mathrm{Se}_{3}=1$. Further

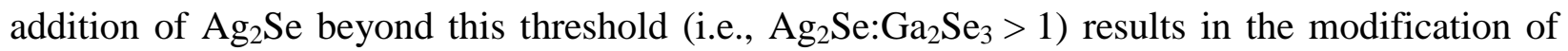
the Ga,Ge-Se tetrahedral network via depolymerization and formation of non-bridging Se atoms. Moreover, all glasses irrespective of their $\mathrm{Ag}_{2} \mathrm{Se}: \mathrm{Ga}_{2} \mathrm{Se}_{3}$ ratio contain a small fraction of Se-Se bonds, implying violation of chemical order. Therefore, the compositional evolution of the structure of these glasses displays the characteristics of both the isoelectronic alkali/alkalineearth aluminosilicate glasses and the purely covalent, continuously alloyed chalcogenide networks. Such composition-dependent structural evolution is shown to be consistent with the corresponding variation in glass transition temperature and molar volume.
\end{abstract}




\section{Introduction}

Multicomponent chalcogenide glasses constitute an important class of materials with technological applications in areas ranging from infrared photonics and telecommunication to non-volatile phase change memory as well as in electrochemical devices as solid electrolytes and chemical sensors [1-4]. An extensive understanding of the structure-property relationship is required in order to optimize the performance of these glasses for their various applications. The majority of structure-property relationship studies in the literature to date have focused on GeAs-P-X $(\mathrm{X}=\mathrm{S}, \mathrm{Se}, \mathrm{Te})$ glasses that are characterized primarily by fully connected covalent networks where constituent atoms follow the 8-N bonding rule [5-10]. In contrast to these network chalcogenide glasses, relatively little is known regarding the structural characteristics and structure-property systematics of chalcogenide glasses with modified networks. From a practical point of view, however, simply modified chalcogenide networks are not good glassformers due to their thermal instability against devitrification and low solubility of alkali and/or alkaline-earth sulfides and selenides in Ge-As-P-X networks [11-15] One way to improve the thermal stability and to incorporate significant amounts of alkali or alkaline-earth modifiers and rare earths in these glasses is to create a compensated network via addition of refractory trivalent cations that are glass-forming intermediates such as $\mathrm{Ga}^{3+}[16-18]$. In this case the modifier cations are expected to charge balance the $\left[\mathrm{Ga}(\mathrm{S} / \mathrm{Se})_{4 / 2}\right]^{-}$tetrahedra and depending on the modifier:intermediate ratio $\mathrm{R}$ one can create a partially or fully compensated network, much like the alkali/alkaline-earth aluminosilicate glasses. These glass compositions are isoelectronic with oxide glasses in the system $\mathrm{M}_{2} \mathrm{O}\left(\mathrm{M}^{\prime} \mathrm{O}\right)-\mathrm{Al}_{2} \mathrm{O}_{3}-\mathrm{SiO}_{2}\left(\mathrm{M}=\right.$ alkali, $\mathrm{M}^{\prime}$ = alkaline-earth $)$ that have been extensively studied in the literature. Since the interatomic bonding is largely covalent in nature in chalcogenides and ionic in oxides, a comparison between the glasses in these systems 
may provide important information on how the structure is controlled by the ionic vs. covalent nature of the constituent bonds. This idea prompted us to recently explore the structure-property relationships in ternary stoichiometric chalcogenide glasses in the systems $\mathrm{Na}_{2} \mathrm{Se}-\mathrm{Ga}_{2} \mathrm{Se}_{3}-\mathrm{GeSe}_{2}$ (NGGS) and BaSe-Ga $\mathrm{Se}_{3}-\mathrm{GeSe}_{2}$ (BGGS) [19-21]. These studies have demonstrated that similar to their oxide counterparts, the modifier:intermediate ratio $\mathrm{R}$ plays an important role in the compositional evolution of the network structure in NGGS and BGGS glasses. In the case of $\mathrm{R}<1$, these glasses can be considered to be Se-deficient for satisfying the tetrahedral coordination of heteropolar bonded Ga and Ge atoms. This Se deficiency is accommodated via the formation of Ge-Ge homopolar bonds in the structure. On the other hand, for R > 1 there are strong indications for the formation of non-bridging Se (NBSe) atoms, although a direct and unequivocal experimental observation of such species remains elusive. Recently we have extended such studies to the system $\mathrm{Ag}_{2} \mathrm{Se}_{-} \mathrm{Ga}_{2} \mathrm{Se}_{3}-\mathrm{GeSe}_{2}$ (AGGS) where we have reported the formation of homogeneous glasses and $\mathrm{Ag}$ was shown to behave as a modifier cation with significant mobility leading to superionic conduction even at ambient temperature [22]. In the present study, we report the results of a detailed investigation of the homogeneous glass-forming region and structural characteristics of glasses in the AGGS system using Raman and ${ }^{77} \mathrm{Se},{ }^{69} \mathrm{Ga}$, and ${ }^{71} \mathrm{Ga}$ nuclear magnetic resonance (NMR) spectroscopic techniques. The compositional evolution of the structure of these glasses is linked to the corresponding variation in molar volume and glass transition temperature $T_{g}$. 


\section{Experimental Methods}

\subsection{Synthesis}

All AGGS glasses were synthesized by conventional melt quenching method. The glasses were prepared in $2 \mathrm{~g}$ batches from mixtures of constituent elements with $99.9999 \%$ purity level. The mixtures were sealed in evacuated $\left(\sim 10^{-4}\right.$ Torr $)$ fused silica ampoules and melted in a rocking furnace. The mixtures were heated to $1050{ }^{\circ} \mathrm{C}$ at a rate of $100{ }^{\circ} \mathrm{C} / \mathrm{h}$, held at temperature for $6 \mathrm{~h}$ and were subsequently cooled to $950{ }^{\circ} \mathrm{C}$ and held at temperature for 24 hours followed by quenching in ice water. The amorphous nature of the samples was verified using powder X-ray diffraction measurements.

\subsection{Raman Spectroscopy}

The Raman spectra of the AGGS glasses were collected using a Bruker RFS 100/S Fourier-transform (FT) Raman spectrometer equipped with a Nd:YAG laser operating at a wavelength of $1064 \mathrm{~nm}$. Each Raman spectrum is the average of 64 to 128 scans collected in backscattering geometry using a resolution of $2 \mathrm{~cm}^{-1}$ and a laser power of $30 \mathrm{~mW}$.

\subsection{NMR Spectroscopy}

\subsubsection{Experiments at high magnetic field (19.6 T)}

All experiments at the field of $19.6 \mathrm{~T}$ were performed at the National High Magnetic Field Laboratory using an ultra-narrow bore magnet equipped with a Bruker DRX console operating at resonance frequencies of $158.8,253.6$ and $199.7 \mathrm{MHz}$ for ${ }^{77} \mathrm{Se},{ }^{71} \mathrm{Ga}$ and ${ }^{69} \mathrm{Ga}$, respectively. High-resolution two-dimensional (2D) ${ }^{71} \mathrm{Ga}$ quadrupolar magic-angle-turning (QMAT) and ${ }^{77} \mathrm{Se}$ magic-angle-turning phase-adjusted sideband separation (MATPASS) 
experiments were performed with Car-Purcell-Meiboom-Gill (CPMG) echo-train acquisition $[23,24]$. Crushed samples were packed in $4 \mathrm{~mm} \mathrm{ZrO}_{2}$ rotors and spun at $10 \mathrm{kHz}$. For each ${ }^{71} \mathrm{Ga}$ and ${ }^{69} \mathrm{Ga} 2 \mathrm{D}$ QMAT/CPMG experiment 64 hypercomplex $t_{1}$ points were acquired with 2000 (6000 for ${ }^{69} \mathrm{Ga}$ ) transients per point, $33 \mathrm{CPMG}$ echoes per transient and a 0.5 s recycle delay. The details of the QMAT/CPMG pulse sequence can be found elsewhere [22,23]. Each transient was obtained using the MAT sequence of nine $\pi$-pulses. The MAT sequence is followed by CPMG pulses for multiple-echo acquisition. The $\pi / 2$ - and $\pi$-pulse lengths were 2.0 and $4.0 \mu \mathrm{s}$, respectively. The ${ }^{77}$ Se 2D MATPASS/CPMG experiment used a series of five MAT $\pi$-pulses (4.0 $\mu \mathrm{s})$ followed by CPMG pulses for data acquisition. Each of the 16 hypercomplex $t_{1}$ points were acquired with 36 transients per point, $64 \mathrm{CPMG}$ echoes per transient, and a $60 \mathrm{~s}$ recycle delay. The inter $\pi$-pulse delays for both QMAT and MATPASS experiments were incremented according to the timings detailed by Hung et al. [24]. Hypercomplex data acquisition was employed for both QMAT and MATPASS experiments, using the method of States et al. [25] to the phases of the CPMG pulses and the receiver. Referencing for ${ }^{71} \mathrm{Ga} /{ }^{69} \mathrm{Ga}$ and ${ }^{77} \mathrm{Se}$ was done externally by recording the ${ }^{17} \mathrm{O}$ signal of neat, natural abundance $\mathrm{H}_{2} \mathrm{O}$ and then using the frequency ratios reported in the IUPAC recommendations [26].

\subsubsection{Experiments at low magnetic field (11.7 T)}

All NMR measurements at $11.7 \mathrm{~T}$ were performed using a Bruker AQS spectrometer operating at $95.4 \mathrm{MHz}$ for ${ }^{77} \mathrm{Se}$. The ${ }^{77} \mathrm{Se}$ magic angle spinning (MAS) experiments were performed using a $5 \mathrm{~mm}$ Doty XC probe with crushed glass samples spinning in a $\mathrm{Si}_{3} \mathrm{~N}_{4}$ rotor at $\sim 15 \mathrm{kHz}$. A Hahn echo pulse sequence was employed with a rotor synchronized delay between the $\pi / 2(2.2 \mu \mathrm{s})$ and $\pi(4.4 \mu \mathrm{s})$ pulses and a recycle delay of $200 \mathrm{~s}$. Approximately 900 FIDs were 
averaged and Fourier transformed to obtain each spectrum. The ${ }^{77} \mathrm{Se}$ chemical shifts were referenced to the ${ }^{77} \mathrm{Se}$ isotropic shift of crystalline $\left(\mathrm{NH}_{4}\right)_{2} \mathrm{SeO}_{4}\left(\delta_{\text {iso }}=1040.2 \mathrm{ppm}\right)$. All simulations of the NMR spectra were performed using the Dmfit software package [27]. The reported chemical shift anisotropy (CSA) tensors follow the Haeberlen convention defined as:

$\delta_{z z}-\delta_{i s o} \geq \delta_{x x}-\delta_{y y} \geq \delta_{y y}-\delta_{i s o}$,

$\delta_{i s o}=\frac{1}{3}\left(\delta_{z z}+\delta_{x x}+\delta_{y y}\right)$,

$\Delta=\delta_{z z}-\delta_{i s o}$,

$\eta=\frac{\delta_{x x}-\delta_{y y}}{\Delta}$,

where $\delta_{\mathrm{xx}}, \delta_{\mathrm{yy}}$, and $\delta_{z z}$ are the principal components of the CSA tensor. The magnitude of the CSA is $\Delta$, while the asymmetry of the CSA is denoted by $\eta$.

\subsection{Differential Scanning Caloriemetry (DSC)}

Conventional DSC scans were taken using a Mettler-Toledo DSC1 calorimeter. Scans were performed in a flowing nitrogen environment on 10-20 mg of sample loaded into $40 \mu \mathrm{L}$ aluminum crucibles. The glass transition temperature $T_{g}$ is determined to within $\pm 2^{\circ} \mathrm{C}$ as the onset of the glass transition region, when heating at a rate of $20^{\circ} \mathrm{C} / \mathrm{min}$.

\subsection{Density measurements}

Density measurements were carried out using a Micromeritics AccuPyc II gas expansion pycnometer under a helium environment of $6 \mathrm{~N}$ purity. Approximately 1 to $2 \mathrm{~g}$ of each sample was loaded into a $1 \mathrm{~cm}^{3}$ cup. Reported densities are averages of 10 consecutive measurements at $20{ }^{\circ} \mathrm{C}$ and are determined to within $\pm 0.005 \mathrm{~g} / \mathrm{cm}^{3}$. 


\section{Results}

\subsection{Glass Formation Range}

The homogeneous glass formation range is shown in Fig. 1. Previous studies reported the formation of homogeneous binary glasses along the $\mathrm{Ga}_{2} \mathrm{Se}_{3}-\mathrm{GeSe}_{2}$ join with 0 to $30 \mathrm{~mol} \%$ $\mathrm{Ga}_{2} \mathrm{Se}_{3}$ [28]. At least $10 \mathrm{~mol} \% \mathrm{Ga}_{2} \mathrm{Se}_{3}$ is required to form a homogeneous ternary glass with $\mathrm{Ag}_{2} \mathrm{Se}$ as a component with up to $40 \mathrm{~mol} \% \mathrm{Ag}_{2} \mathrm{Se}$. On the other hand, glass formation for

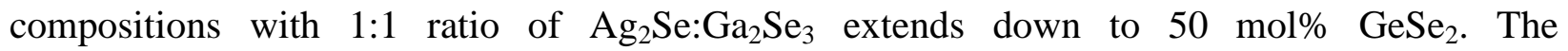
homogeneous glass formation range is surrounded by a narrow composition region where crystallization can still be avoided at typical cooling rates but the resulting glasses are heterogeneous, i.e., phase separated (Fig. 1). These phase separated glasses are characterized by two distinct glass transitions in the DSC scans (Fig. 2). For example, the ternary phase separated glass of composition $\left(\mathrm{Ag}_{2} \mathrm{Se}\right)_{10}\left(\mathrm{Ga}_{2} \mathrm{Se}_{3}\right)_{40}\left(\mathrm{GeSe}_{2}\right)_{50}$ displays two glass transitions that are situated above and below the single glass transition observed for the $\left(\mathrm{Ag}_{2} \mathrm{Se}\right)_{25}\left(\mathrm{Ga}_{2} \mathrm{Se}_{3}\right)_{25}\left(\mathrm{GeSe}_{2}\right)_{50}$ glass (Fig. 2). The heterogeneous glass is therefore, likely separating into a low- $\mathrm{T}_{\mathrm{g}} \mathrm{Ag}$ rich glass in the matrix of a high- $\mathrm{T}_{\mathrm{g}}$ glass composed primarily of GaGe-Se, given the similarity between the higher $T_{g}$ value in comparison with those characteristic of the binary $\mathrm{Ga}_{2} \mathrm{Se}_{3}-\mathrm{GeSe}_{2}$ glasses [28]. This type of phase separation has been observed before in the binary $\mathrm{Ag}_{2} \mathrm{Se}_{-}-\mathrm{GeSe}_{2}$ glass system [29] and a representative case is shown in Fig. 2 for comparison. As the extent of phase separation in these glasses is difficult to determine, here we use the criterion that glasses that lack a detectable second glass transition are homogeneous and the structure-property relations in these glasses are the focus of the present study. Liquids in the composition region surrounding the homogeneous and heterogeneous glass formation devitrify readily upon cooling (Fig. 1). All of the bulk glasses are a lustrous dark grey in color, however, 
increasing the $\mathrm{Ag}$ :Ga ratio changes the color of the crushed glass from a dark reddish orange to black for the $\mathrm{R}>1$ glasses indicating a redshift of the optical absorption edge. The compositions of the homogeneous AGGS glasses prepared in this study and their $\mathrm{T}_{\mathrm{g}}$ and density are listed in Table 1.

\subsection{Raman Spectroscopy}

The Raman spectra of all AGGS glasses are shown in Fig. 3. All spectra display three distinct regions: (i) a broad band from $\sim 60$ to $160 \mathrm{~cm}^{-1}$, (ii) a sharp peak at $200 \mathrm{~cm}^{-1}$ with shoulders near $\sim 175$ and $\sim 215 \mathrm{~cm}^{-1}$, and (iii) a broad band centered at $\sim 250$ to $263 \mathrm{~cm}^{-1}$ with a shoulder at $\sim 305$ $\mathrm{cm}^{-1}$. The broad band in region (i) contains various bending modes of the $\mathrm{GeSe}_{4 / 2}$ tetrahedra. Previous studies have assigned the primary band at $200 \mathrm{~cm}^{-1}$ in region (ii) to the $A_{I}$ symmetric stretching of the corner sharing $\mathrm{GeSe}_{4 / 2}$ tetrahedra [30]. The shoulders at 175 and $215 \mathrm{~cm}^{-1}$ are assigned to the stretching of homopolar Ge-Ge bonds in an ethane like unit $\left(\mathrm{Se}_{3}\right) \mathrm{Ge}-\mathrm{Ge}\left(\mathrm{Se}_{3}\right)$ and of Ge-Se bonds in edge-shared $\mathrm{GeSe}_{4 / 2}$ tetrahedra, respectively. The main band in region (iii) near $\sim 250$ to $260 \mathrm{~cm}^{-1}$ corresponds to the stretching mode of homopolar Se-Se bonds and a coupled bending and asymmetric stretching mode of the $\mathrm{GeSe}_{4 / 2}$ tetrahedra while the shoulder near $305 \mathrm{~cm}^{-1}$ corresponds to the asymmetric stretching of the GeSe $4 / 2$ tetrahedra [19]. A sharp but weak peak at $80 \mathrm{~cm}^{-1}$ from the sample holder can be discerned in some spectra.

Changes in the Raman spectra for the $R>1$ glasses are shown in Fig. 3 (a) where the concentration of $\mathrm{Ag}_{2} \mathrm{Se}$ is increased relative to $\mathrm{GeSe}_{2}$ along a fixed concentration of $10 \mathrm{~mol} \%$ $\mathrm{Ga}_{2} \mathrm{Se}_{3}$. The most prominent change with increasing $\mathrm{Ag}_{2} \mathrm{Se}$ is the decreasing intensity of the shoulder near $215 \mathrm{~cm}^{-1}$ indicating the disappearance of the edge-sharing $\mathrm{GeSe}_{4 / 2}$ tetrahedra in the glass structure and a consequent shift to a predominantly corner-sharing network. Additionally 
the peak position of the high-frequency band shifts progressively with increasing $\mathrm{Ag}_{2} \mathrm{Se}$ from $\sim 261 \mathrm{~cm}^{-1}$ to $\sim 252 \mathrm{~cm}^{-1}$. This shift has been attributed in the literature to the increased involvement of non-bridging Se on the $\mathrm{GeSe}_{4 / 2}$ tetrahedra forming Ge-Se-Ag linkages [31].

The Raman spectra for the $\mathrm{R}=1$ glasses are shown in Fig. 3 (b) as a function of the total $\mathrm{GeSe}_{2}$ content. The compositional evolution shows significant asymmetric broadening of the main mode at $\sim 200 \mathrm{~cm}^{-1}$ and increasing intensity near $175 \mathrm{~cm}^{-1}$ as $\mathrm{GeSe}_{2}$ content decreases. At the same time the definition of the $215 \mathrm{~cm}^{-1}$ band disappears and the band at $\sim 260 \mathrm{~cm}^{-1}$ broadens and shifts slightly to lower wavenumbers with decreasing $\mathrm{GeSe}_{2}$ content.

Fig. 3 (c) shows the Raman spectra for the $\mathrm{R}<1$ glasses with a narrow range of 7.5 to $10 \mathrm{~mol} \%$ $\mathrm{Ag}_{2} \mathrm{Se}$, where the $\mathrm{Ga}_{2} \mathrm{Se}_{3}$ content is increased relative to $\mathrm{GeSe}_{2}$. In contrast to the other series of glasses, increasing the $\mathrm{Ga}$ content has no effect on region (iii). In region (ii) there is a pronounced increase in the intensity of the $175 \mathrm{~cm}^{-1}$ band indicating the formation of homopolar Ge-Ge bonds. As discussed in section 3.4, the ${ }^{71}$ Ga NMR spectroscopic results indicate that Ga atoms do not participate significantly in homopolar bonding in these glasses. The increased number of homopolar Ge-Ge bonds results from Se deficiency as $\mathrm{Ga}$ is added in the stoichiometric form of $\mathrm{GaSe}_{3 / 2}$, thus lacking enough $\mathrm{Se}$ atoms to satisfy a fourfold coordination for $\mathrm{Ga}$ atoms in the glass structure [32].

\section{$3.3{ }^{77} \mathrm{Se} N \mathrm{NMR}$}

The ${ }^{77}$ Se MAS NMR spectra of AGGS glasses with 10 mol\% $\mathrm{Ga}_{2} \mathrm{Se}_{3}$ and with $\mathrm{Ag}_{2} \mathrm{Se}$ content varying between 16 and $40 \mathrm{~mol} \%$ are shown in Fig. 4. These spectra consist of a main peak centered at $\sim 390 \mathrm{ppm}$, characteristic of two-coordinated Se sites corner-shared by $(\mathrm{Ge}, \mathrm{Ga}) \mathrm{Se}_{4}$ tetrahedra [32]. However, these spectral line shapes cannot be simulated well with a single 
Gaussian peak at this position. Excess intensity is apparent in the form of a high-frequency shoulder near $700 \mathrm{ppm}$ and a low-frequency shoulder near $0 \mathrm{ppm}$. Although the intensity of the high-frequency shoulder appears to be independent of the Ag concentration, intensity on the lowfrequency side increases with increasing Ag content (Fig. 4). A 2D ${ }^{77} \mathrm{Se}$ MATPASS/CPMG NMR spectrum was collected on the sample with $40 \mathrm{~mol} \% \mathrm{Ag}_{2} \mathrm{Se}$ (Fig. 5a) in order to gain further insight on the constituent NMR line shapes under the MAS spectra in Fig. 4. The isotropic projection of a MATPASS/CPMG NMR spectrum is free of CSA broadening effects. This projection for the ${ }^{77}$ Se MATPASS/CPMG NMR spectrum in Fig. 5a is shown in Fig. 5b. It can be simulated well with three Gaussian peaks with $\delta_{\text {iso }}$ centered near 550, 375 and $130 \mathrm{ppm}$ with relative fractions of 5, 50 and $45 \%$, respectively. The corresponding CSA tensors are obtained from simulating the spinning sidebands in the corresponding projections on the anisotropic dimension (Fig. 5c). The simulation for the site with $\delta_{\text {iso }}=550 \mathrm{ppm}$ (375 ppm) yields a CSA tensor with $\Delta=290 \mathrm{ppm}(\Delta=250 \mathrm{ppm})$ and $\eta=0.85(\eta=0.9) . \quad$ These $\delta_{\text {iso }}$ and CSA values are consistent with the assignment of these two peaks to Ge-Se-Se and cornersharing Ge-Se-Ge environments in the glass structure, respectively [33]. In contrast, the peak with $\delta_{\text {iso }} \sim 130 \mathrm{ppm}$ is characterized by a significantly larger $\Delta$ of $\sim-450 \mathrm{ppm}$ and $\eta=0.7$. This peak can be assigned on the basis of its $\delta_{\text {iso }}$ value to non-bridging Se (NBSe) atoms in Ge-Se-Ag environments of the glass structure [22]. The relatively large $\Delta$ associated with this Se environment is also consistent with the structural and chemical symmetry of this environment. The $\delta_{\text {iso }}$ and CSA parameters obtained from the ${ }^{77}$ Se MATPASS NMR spectrum allows for a fairly accurate simulation of the MAS spectral line shapes of the AGGS glasses, even when the peak positions and their full widths at half maximum are kept constrained. These simulations are shown in Fig. 4 and the corresponding parameters are listed in Table 2. The results of these 
simulations directly show the progressively increasing concentration of NBSe environments in AGGS glasses with increasing Ag concentration. The total fraction of NBSe environments as obtained from these simulations is compared in Fig. 6 to the values calculated from the chemical composition of these glasses, under the assumption that the excess Se incorporated via the addition of $\mathrm{Ag}_{2} \mathrm{Se}$ minus that required to satisfy the tetrahedral coordination of Ga goes to form these Se environments. The agreement in the compositional trend between the measured and calculated values indicates that the structural network of the AGGS glasses behaves much like that in charge-compensated aluminosilicate glasses where the modifier cations in excess of those required to charge balance the $\left[\mathrm{AlO}_{4 / 2}\right]^{-1}$ tetrahedra generate non-bridging oxygen atoms. However, the origin of the large offset between the two sets of values remains unclear at this stage. It is likely that the MAS NMR line shape simulations overestimate the fraction of NBSe environments as the corresponding signal may contain overlapping contributions from other types of bridging Se sites. This hypothesis is corroborated by the fact that for the sample with 40 mol\% $\mathrm{Ag}_{2} \mathrm{Se}$, the NBSe fraction obtained from the ${ }^{77} \mathrm{Se}$ MATPASS/CPMG NMR spectrum $(45 \%)$ is $\sim 5 \%$ lower than that obtained from the simulation of the corresponding MAS spectrum (see Table 2).

\section{$3.4^{71 / 69}$ Ga QMAT/CPMG NMR}

The ${ }^{71} \mathrm{Ga}$ central transition QMAT/CPMG NMR spectra for three representative glasses with $\mathrm{R}<1, \mathrm{R}=1$, and $\mathrm{R}>1$ are shown in Fig. 7. The $\mathrm{R}<1$ and $\mathrm{R}=1\left(32.5 \%\right.$ and $20 \% \mathrm{Ga}_{2} \mathrm{Se}_{3}$ respectively) glasses in Fig. 7a display a single asymmetric peak that exhibits a maximum near $130 \mathrm{ppm}$ and a low frequency tail that is typical of MAS NMR spectra of quadrupolar nuclides in 
disordered materials where it is associated with a distribution of quadrupolar coupling constants, $C_{Q}$. Simulation of these lineshapes using the Czjzek model of distribution of $C_{Q}$ in the software DMfit [34] yields an isotropic chemical shift $\delta_{\text {iso }} \sim 175 \pm 4 \mathrm{ppm}$ and a quadrupolar coupling constant $C_{Q} \approx 10.5$ to $11.0 \mathrm{MHz}$. These NMR parameters are in good agreement with those reported for the ${ }^{71} \mathrm{Ga}$ MAS NMR spectra corresponding to the tetrahedral $\mathrm{GaSe}_{4}$ environment found in stoichiometric BaSe- $\mathrm{Ga}_{2} \mathrm{Se}_{3}-\mathrm{GeSe}_{2}$ glasses [21]. The ${ }^{71} \mathrm{Ga} \delta_{\text {iso }}$ for these AGGS glasses is also consistent with that reported (130 ppm) for the $\mathrm{Ga}_{2} \mathrm{Se}_{3}$ crystal where $\mathrm{Ga}$ atoms are tetrahedrally coordinated to Se. Hence, it is safe to conclude that the Ga atoms in AGGS glasses with $\mathrm{R} \leq 1$ are predominantly tetrahedrally coordinated to $\mathrm{Se}$ in corner-sharing $\mathrm{GaSe}_{4 / 2}$ environment. It should be noted here that the tetrahedral coordination of $\mathrm{Ga}$ is known to be preserved even in glasses with $\mathrm{R}=0$, i.e., those along the $\mathrm{Ga}_{2} \mathrm{Se}_{3}-\mathrm{GeSe}_{2}$ join [27]. In contrast, the AGGS glasses with $\mathrm{R}>1$ show significantly complicated Ga NMR line shapes (Fig. 7b). Comparison between ${ }^{69} \mathrm{Ga}$ and ${ }^{71} \mathrm{Ga}$ NMR line shapes at $19.6 \mathrm{~T}$ for the glass with $\mathrm{R}=4$ suggest the presence of multiple Ga resonances. In fact these spectra can only be simulated in a selfconsistent manner with contributions from three Ga resonances. The components for the ${ }^{71} \mathrm{Ga}$ NMR spectrum are characterized by sites with $\delta_{\text {iso }} \sim 80,180$ and $250 \mathrm{ppm}$ and $C_{Q} \sim 8,11$ and 9 $\mathrm{MHz}$, respectively. Although the resonance with $\delta_{\text {iso }}=180 \mathrm{ppm}$ and $C_{Q}=11 \mathrm{MHz}$, that constitute $\sim 65 \%$ of the total area under the line shape, can be readily assigned to tetrahedral $\mathrm{GaSe}_{4 / 2}$ environments, the other two resonances cannot be assigned to specific Ga environments a priori. Previous report of ${ }^{71} \mathrm{Ga} \mathrm{NMR}$ results on $\mathrm{GeS}_{2}-\mathrm{P}_{2} \mathrm{~S}_{5}-\mathrm{Ga}_{2} \mathrm{~S}_{3}$ glasses indicated that the $\delta_{\text {iso }}$ for the resonance for octahedrally coordinated Ga shifts to lower frequency (lower ppm) by $\sim 350$ ppm compared to that for tetrahedrally coordinated Ga atoms [35]. Therefore, it may seem reasonable to assign the resonance at $\delta_{\text {iso }} \sim 80 \mathrm{ppm}$ to five-coordinated Ga atoms in AGGS 
glasses with $\mathrm{R}>1$. However, typically network intermediates such as $\mathrm{Ga}$ and $\mathrm{Al}$ are expected to be pushed to higher than fourfold coordination either in glasses deficient in chalcogen atoms or in those with high field strength cations such as phosphorus. Therefore, the formation of higher than fourfold coordinated $\mathrm{Ga}$ atoms in $\mathrm{R}>1$ glasses seems unlikely. On the other hand, formation of NBSe in glasses with $\mathrm{R}>1$ and their association with $\mathrm{Ga}$ in the glass structure may give rise to these additional ${ }^{71} \mathrm{Ga}$ resonances near $\sim 80$ and $250 \mathrm{ppm}$, although the exact nature of such structural units remains unclear at this stage.

\subsection{Differential Scanning Calorimetry}

The compositional variation of $T_{g}$ for all AGGS glasses span a relatively narrow range between 214 and $295{ }^{\circ} \mathrm{C}$ (Table 1). The variation in $\mathrm{T}_{\mathrm{g}}$ with $\mathrm{R}$ for AGGS glasses is shown in Fig. 8a. These glasses show a monotonic decrease in $T_{g}$ with increasing $R$. The variation of $T_{g}$ with $\mathrm{GeSe}_{2}$ content for the $\mathrm{R}=1$ and $\mathrm{R}<1$ glasses is shown in Fig. $8 \mathrm{~b}$. A monotonic decrease in $\mathrm{T}_{\mathrm{g}}$ is observed in all glasses with increasing $\mathrm{GeSe}_{2}$ content.

\subsection{Molar Volume}

The influence of composition on structural packing is best revealed in the molar volume data as the density is dominated by the presence of heavy elements such as Ag. Fig. 9 shows a nearly linear increase in molar volume for all AGGS glasses with increasing $\mathrm{GeSe}_{2}$ content. On the other hand, for comparable $\mathrm{GeSe}_{2}$ contents, molar volume shows small but systematic decrease with increasing R (Fig. 9). 


\section{Discussion}

When taken together, the Raman and NMR spectroscopic results indicate that the structure of the AGGS glasses is primarily based on a corner sharing network of $(\mathrm{Ge} / \mathrm{Ga}) \mathrm{Se}_{4 / 2}$ tetrahedra. Some edge-sharing between these tetrahedra is observed, particularly in glasses with high $\mathrm{GeSe}_{2}$ content. All glasses including those that are Se-deficient (i.e., $\mathrm{R}<1$ ) display the presence of a small concentration of Se-Se linkages, implying violation of chemical order. In glasses with $\mathrm{R}>1$, the $(\mathrm{Ge} / \mathrm{Ga}) \mathrm{Se}_{4 / 2}$ tetrahedral network is modified via formation of NBSe atoms as Ag plays the role of a typical modifier cation. On the other hand, Se-deficient glasses with $\mathrm{R}<1$ display the formation of Ge-Ge homopolar bonds.

A comparison of the modes of structural modification between glasses in the AGGS system studied here and their isoelectronic analogues in alkali or alkaline-earth aluminosilicate system reveals interesting similarities and differences. In both systems, for R $>1$ the presence of excess modifier results in the depolymerization of the tetrahedral network via formation of nonbridging Se or O species. However, the chalcogenide and oxide networks behave differently when $\mathrm{R}<1$. In the oxide system, the network accommodates the oxygen deficiency either by formation of threefold coordinated oxygen atoms while keeping the Al tetrahedrally coordinated or by forcing $\mathrm{Al}$ into a network-modifier like environment in five- and/or six-fold coordination with oxygen [36]. In contrast, in the AGGS system, the Ga remains tetrahedrally coordinated but the Se deficiency is accommodated in the network via formation of Ge-Ge homopolar bonds. Similar observation was also made recently in chalcogenide glasses with modified and compensated networks in $\mathrm{BaSe}-\mathrm{Ga}_{2} \mathrm{Se}_{3}-\mathrm{GeSe}_{2}$ and $\mathrm{Na}_{2} \mathrm{Se}_{-}-\mathrm{Ga}_{2} \mathrm{Se}_{3}-\mathrm{GeSe}_{2}$ systems [20, 21].

This compositional evolution of the atomic structure of AGGS glasses is consistent with the concomitant variation in $\mathrm{T}_{\mathrm{g}}$ as shown in Fig. 8 that display two major trends. One of these is 
the monotonic drop in $\mathrm{T}_{\mathrm{g}}$ with increasing $\mathrm{R}$ at a fixed $\mathrm{Ga}_{2} \mathrm{Se}_{3}$ concentration (Fig. 7a). This trend in glasses with $\mathrm{R} \geq 1$ can be associated with the progressive depolymerization of the tetrahedral network via the formation of NBSe environments. On the other hand, for $\mathrm{R}<1$ such a trend can be explained by considering the Raman and ${ }^{77} \mathrm{Se}$ NMR spectroscopic results, both of which indicate formation of weak Se-Se linkages and violation of chemical order as $\mathrm{Ag}_{2} \mathrm{Se}$ is added to binary $\mathrm{Ga}_{2} \mathrm{Se}_{3}-\mathrm{GeSe}_{2}$ glasses. The other trend is that at constant $\mathrm{R}, \mathrm{T}_{\mathrm{g}}$ decreases with increasing concentration of $\mathrm{GeSe}_{2}$. This trend is consistent with the fact that for comparable degrees of network connectivity, i.e., similar R values, the replacement of Ge-Se bonds with Ga-Se bonds would be expected to give rise to a stronger network as the dissociation energy of the Ga-Se bond is significantly higher than that of the Ge-Se bonds [37, 38]. Similar compositional trends were also reported for analogous $\mathrm{Na}_{2} \mathrm{Se}_{-} \mathrm{Ga}_{2} \mathrm{Se}_{3}-\mathrm{GeSe}_{2}$ glasses in a previous study [20].

Similar to $\mathrm{T}_{\mathrm{g}}$, the molar volume also appears to be controlled in a systematic fashion as a function of $\mathrm{GeSe}_{2}$ content and $\mathrm{R}$. The decrease in molar volume with increasing $\mathrm{R}$ is consistent with the progressive depolymerization of the open tetrahedral network via formation of NBSe which allows for efficient structural packing via collapse of the network. Similar effect of nonbridging oxygen on the packing of aluminosilicate networks is well documented in the literature [39]. On the other hand, the relatively rapid increase in molar volume with increasing $\mathrm{GeSe}_{2}$ content clearly suggests that the tetrahedral $\mathrm{GeSe}_{2}$ network is significantly more open compared to the charge compensated network formed by the $\mathrm{Ag}^{+}\left[\mathrm{GaSe}_{4 / 2}\right]^{-}$units. This trend is quite the opposite of that observed in alkali aluminosilicate liquids where the molar volume at constant $\mathrm{R}$ increases with decreasing $\mathrm{SiO}_{2}$ content [37].

\section{Conclusions}


The structure-property relationship in homogeneous AGGS glasses in the ternary system $\mathrm{Ag}_{2} \mathrm{Se}$ $\mathrm{Ga}_{2} \mathrm{Se}_{3}-\mathrm{GeSe}_{2}$ is investigated. The results of Raman and multinuclear NMR spectroscopy indicate that the structure of these glasses is based on a $(\mathrm{Ga} / \mathrm{Ge}) \mathrm{Se}_{4 / 2}$ tetrahedral network where Ag plays the role of a conventional network modifier in glasses with $\mathrm{R}>1$. This role of $\mathrm{Ag}$ is manifested in the progressively increasing concentration of NBSe species and a monotonic decrease in $\mathrm{T}_{\mathrm{g}}$ upon increasing $\mathrm{R}$ beyond its threshold value of 1 . In contrast, glasses with $\mathrm{R}<1$ display the formation of Ge-Ge homopolar bonds resulting from the deficiency in Se required to satisfy the tetrahedral coordination requirements for both $\mathrm{Ga}$ and Ge atoms. At the same time a small fraction of Se-Se homopolar bonds is observed in all glasses investigated in this study, irrespective of their $\mathrm{R}$ value. The formation of these weak homopolar bonds upon addition of $\mathrm{Ag}$ is responsible for a drop in $T_{g}$ in AGGS glasses with $R<1$. For comparable $R$ values, $T_{g}$ decreases with increasing $\mathrm{GeSe}_{2}$ content which appears to be related to the replacement of stronger Ga-Se bonds with weaker Ge-Se bonds in the tetrahedral network. The effect of R on molar volume appears to be rather subtle as the latter is primarily controlled by the partial molar volume of the open tetrahedral network of the $\mathrm{GeSe}_{2}$ component.

\section{Acknowledgements}

This work was supported by a grant from the National Science Foundation (NSF-DMR 1104869) to SS. 


\section{References}

1. Eggleton, B.J., B. Luther-Davies, and K. Richardson, Chalcogenide photonics. Nat Photon, 2011. 5(3): p. 141-148.

2. Sousa, V., Chalcogenide materials and their application to Non-Volatile Memories. Microelectronic Engineering, 2011. 88(5): p. 807-813. DOI: 10.1016/j.mee.2010.06.042.

3. Tatsumisago, M., Glassy materials based on Li2S for all-solid-state lithium secondary batteries. Solid State Ionics, 2004. 175(1-4): p. 13-18. DOI: 10.1016/j.ssi.2004.09.012.

4. Vassilev, V.S. and S.V. Boycheva, Chemical sensors with chalcogenide glassy membranes. Talanta, 2005. 67(1): p. 20-7. DOI: 10.1016/j.talanta.2005.02.027.

5. Yang, G., et al., Physical properties of the GexSe1-x glasses in the $0<x<0.42$ range in correlation with their structure. Journal of Non-Crystalline Solids, 2013. 377: p. 54-59. DOI: 10.1016/j.jnoncrysol.2013.01.049.

6. Bytchkov, A., et al., Unraveling the atomic structure of Ge-rich sulfide glasses. Physical chemistry chemical physics : PCCP, 2013. 15(22): p. 8487-94. DOI: $10.1039 / \mathrm{c} 3 \mathrm{cp} 50536 \mathrm{~g}$.

7. Giridhar, A. and S. Mahadevan, The Tg versus Z dependence of glasses of the Ge-In-Se system. Journal of Non-Crystalline Solids, 1992. 151: p. 245-252.

8. Sen, S., et al., Structure, topology and chemical order in Ge-As-Te glasses: a high-energy $x$-ray diffraction study. Journal of physics. Condensed matter : an Institute of Physics journal, 2010. 22(40): p. 405401. DOI: 10.1088/0953-8984/22/40/405401.

9. Gjersing, E.L., S. Sen, and B.G. Aitken, Structure, Connectivity, and Configurational Entropy of GexSe100-x Glasses: Results from 77Se MAS NMR Spectroscopy. Journal of Physical Chemistry C, 2010. 114(18): p. 8601-8608. 
10. Yang, G., et al., Correlation between structure and physical properties of chalcogenide glasses in theAsxSe1-xsystem. Physical Review B, 2010. 82(19). DOI: 10.1103/PhysRevB.82.195206.

11. Ribes, M., B. Barrau, and J.L. Souquet, Sulfide glasses: glass forming region, structure and ionic conduction of glasses in Na2S-XS2 (X=Si; Ge), Na2S-P2S5 and Li2S-GeS2 systems. Journal of Non-Crystalline Solids, 1980. 38-39: p. 271-276.

12. Pradel, A., et al., 29Si NMR structural studies of ionically conductive silicon chalcogenide glasses and model compounds. Journal of Non-Crystalline Solids, 1995. 188: p. $75-86$.

13. Bischoff, C., et al., IR, Raman, and NMR studies of the short-range structures of $0.5 N a 2 S$ $+0.5[x G e S 2+(1-x) P S(5 / 2)]$ mixed glass-former glasses. The journal of physical chemistry. B, 2014. 118(7): p. 1943-53. DOI: 10.1021/jp4111053.

14. Mutolo, P.F., et al., Nuclear magnetic resonance (NMR) studies of the local structure of phosphorus chalcogenide glasses: an overview. Journal of Non-Crystalline Solids, 1999. 256 \& 257: p. 63-72.

15. Stehlik, S., et al., Phase Separation in Chalcogenide Glasses: The System AgAsSSe. International Journal of Applied Glass Science, 2011. 2(4): p. 301-307. DOI: 10.1111/j.2041-1294.2011.00065.x.

16. Calvez, L., et al., Influence of gallium and alkali halide addition on the optical and thermo-mechanical properties of GeSe2-Ga2Se3 glass. Applied Physics A, 2007. 89(1): p. 183-188. DOI: $10.1007 /$ s00339-007-4081-y. 
17. Yao, W., K. Berg, and S. Martin, Structure and properties of glasses in the $M I+M 2 S+(0.1 G a 2 S 3+0.9 G e S 2), M=L i, N a, K$ and $C s$, system. Journal of NonCrystalline Solids, 2008. 354(18): p. 2045-2053. DOI: 10.1016/j.jnoncrysol.2007.11.006.

18. Harbison, B.B., C.I. Merzbacher, and I.D. Aggarwal, Preparation and properties of BaSGa2S3-GeS2 glasses. Journal of Non-Crystalline Solids, 1997. 213 \& 214: p. 16-21.

19. Mao, A.W., B.G. Aitken, and S. Sen, Synthesis and physical properties of chalcogenide glasses in the system BaSe-Ga2Se3-GeSe2. Journal of Non-Crystalline Solids, 2013. 369: p. 38-43. DOI: 10.1016/j.jnoncrysol.2013.03.020.

20. Mao, A.W., et al., Synthesis and characterization of ternary glasses in the system Na2SeGa2Se3-GeSe2. Journal of Non-Crystalline Solids, 2014. 404: p. 91-97. DOI: 10.1016/j.jnoncrysol.2014.07.042.

21. Mao, A.W., et al., Structure and bonding characteristics of chalcogenide glasses in the system BaSeGa2Se3GeSe2. Journal of Non-Crystalline Solids, 2013. 375: p. 40-46. DOI: 10.1016/j.jnoncrysol.2013.04.061.

22. Marple, M., et al., Superionic Conduction of Silver in Homogeneous Chalcogenide Glasses. J. Mater. Chem. A, 2015. DOI: 10.1039/C5TA07301D

23. Hung, I. and Z. Gan, A magic-angle turning NMR experiment for separating spinning sidebands of half-integer quadrupolar nuclei. Chemical Physics Letters, 2010. 496(1-3): p. 162-166. DOI: 10.1016/j.cplett.2010.07.016.

24. Hung, I., et al., MATPASS/CPMG: a sensitivity enhanced magic-angle spinning sideband separation experiment for disordered solids. Journal of magnetic resonance, 2012. 221: p. 103-9. DOI: 10.1016/j.jmr.2012.05.013. 
25. States, D.J., R.A. Haberkorn, and D.J. Ruben, A Two-Dimensional Nuclear Overhauser Experiment with Pure Absorption Phase in Four Quadrants. Journal of magnetic resonance, 1982. 48: p. 286-292.

26. Harris, R.K.B., E. D.; De Menezes, S. M. C.; Goodfellow, R.; Granger, P, NMR nomenclature. Nuclear spin properties and conventions for chemical shifts(IUPAC Recommendations 2001). Pure and Applied Chemistry, 2001. 73(11): p. 1795.

27. Massiot, D., et al., Modelling one- and two-dimensional solid-state NMR spectra. Magnetic Resonance in Chemistry, 2002. 40(1): p. 70-76. DOI: 10.1002/mrc.984.

28. Mao, A.W., et al., Structure of glasses in the pseudobinary system $\mathrm{Ga}(2) \mathrm{Se}(3)-\mathrm{GeSe}(2)$ : violation of chemical order and 8 - $N$ coordination rule. The journal of physical chemistry. B, 2013. 117(51): p. 16594-601. DOI: 10.1021/jp410017k.

29. Mitkova, M., Y. Wang, and P. Boolchand, Dual chemical role of Ag as an additive in chalcogenide glasses. Physical review letters, 1999. 83(19): p. 3848.

30. Němec, P., B. Frumarová, and M. Frumar, Structure and properties of the pure and Pr 3+-doped Ge $25 \mathrm{Ga} 5 \mathrm{Se} 70$ and $\mathrm{Ge} 30 \mathrm{Ga} 5$ Se 65 glasses. Journal of non-crystalline solids, 2000. 270(1): p. 137-146.

31. Le Parc, R., et al., Ag-Ge-Se glasses: a vibrational spectroscopy study. Journal of Raman Spectroscopy, 2013. 44(7): p. 1049-1057. DOI: 10.1002/jrs.4320.

32. Mao, A.W., et al., Mechanisms of structural accommodation of Se deficiency in binary Ga2Se3-GeSe2 glasses: Results from 77Se MATPASS/CPMG NMR spectroscopy. Journal of Non-Crystalline Solids, 2015. 410: p. 14-19. DOI: 10.1016/j.jnoncrysol.2014.12.003. 
33. Kaseman, D.C., et al., Observation of a continuous random network structure in Ge(x)Se(100-x) glasses: results from high-resolution 77Se MATPASS/CPMG NMR spectroscopy. The journal of physical chemistry. B, 2013. 117(3): p. 949-54. DOI: 10.1021/jp311320t.

34. Massiot, D., et al., Modelling One-and Two-Dimensional Solid-State NMR Spectra. Magn. Reson. Chem., 2002. 40(1): p. 70-76. DOI: 10.1002/mrc.984.

35. Youngman, R.E. and B.G. Aitken, Structure and properties of GeGaP sulfide glasses. Journal of Non-Crystalline Solids, 2004. 345-346: p. 50-55. DOI:

10.1016/j.jnoncrysol.2004.07.042.

36. Zirl, D.M. and S.H. Garofalini, Structure of Sodium Aluminosilicate Glasses. Journal of the American Ceramic Society, 1990. 73(10): p. 2848-2856.

37. Nedeva, Y., et al., Compositional dependance of the optical properties of the Ge-Se-Ga glasses. Journal of Optoelectronics and Advanced Materials, 2001. 3(2): p. 433-436.

38. Afifi, M.A., et al., Electrical and thermal properties of chalcogenide glass system Se75Ge25-xSbx. Applied Physics A, 1992. 55: p. 167-169.

39. Mysen, B. and P. Richet, Silicate glasses and melts: properties and structure. Vol. 10. 2005: Elsevier. 
Table 1: Composition (mol\%), $\mathrm{T}_{\mathrm{g}}, \mathrm{T}_{\mathrm{x}}$ (crystallization temperature), and density for all homogeneous AGGS glasses synthesized in this study.

\begin{tabular}{|c|c|c|c|c|c|}
\hline $\mathbf{A g}_{2} \mathbf{S e}$ & $\mathbf{G a}_{2} \mathbf{S e}_{3}$ & $\mathbf{G e S e}_{\mathbf{2}}$ & $\mathbf{T}_{\mathbf{g}}\left({ }^{\circ} \mathbf{C}\right)$ & $\mathbf{T}_{\mathbf{x}}\left({ }^{\circ} \mathbf{C}\right)$ & Density $\left(\mathbf{g ~ c m}^{-3}\right)$ \\
\hline 8 & 10 & 82 & 264 & 379 & 4.588 \\
16 & 10 & 74 & 231 & 344 & 4.934 \\
22 & 10 & 68 & 240 & 337 & 5.076 \\
26 & 10 & 64 & 234 & 325 & 5.214 \\
30 & 10 & 60 & 219 & 328 & 5.345 \\
40 & 10 & 50 & 214 & 312 & 5.502 \\
40 & 15 & 45 & 226 & 336 & 5.633 \\
5 & 5 & 90 & 245 & 375 & 4.397 \\
20 & 20 & 60 & 269 & 328 & 5.067 \\
25 & 25 & 50 & 285 & 349 & 5.224 \\
10 & 20 & 70 & 285 & 366 & 4.726 \\
7.5 & 32.5 & 60 & 295 & 395 & 4.669 \\
\hline
\end{tabular}


Table 2: Line shape simulation parameters for ${ }^{77} \mathrm{Se}$ MAS NMR spectra of the AGGS glass series with $10 \mathrm{~mol} \% \mathrm{Ga}_{2} \mathrm{Se}_{3}$.

\begin{tabular}{|c|c|c|c|c|c|}
\hline$\% \mathrm{Ag}_{2} \mathrm{Se}$ & $\begin{array}{c}\delta_{\text {iso }} \\
( \pm 15 \mathrm{ppm})\end{array}$ & $\begin{array}{l}\text { Width } \\
\text { (ppm) }\end{array}$ & $\begin{array}{c}\Delta \\
(\mathbf{p p m})\end{array}$ & $\eta$ & $\begin{array}{l}\text { relative } \\
\text { fraction } \\
( \pm 10 \%)\end{array}$ \\
\hline \multicolumn{6}{|l|}{$16 \%$} \\
\hline Ge-Se-Ge & 390 & 240 & 250 & 0.9 & 51.64 \\
\hline Ge-Se-Ag & 130 & 240 & -450 & 0.7 & 27.80 \\
\hline Se-Se-Ge & 570 & 240 & 290 & 0.85 & 20.56 \\
\hline \multicolumn{6}{|l|}{$26 \%$} \\
\hline Ge-Se-Ge & 388 & 240 & 250 & 0.9 & 44.87 \\
\hline Ge-Se-Ag & 130 & 240 & -450 & 0.7 & 37.80 \\
\hline Se-Se-Ge & 570 & 240 & 290 & 0.85 & 17.33 \\
\hline \multicolumn{6}{|l|}{$40 \%$} \\
\hline Ge-Se-Ge & 390 & 240 & 250 & 0.9 & 39.16 \\
\hline Ge-Se-Ag & 130 & 240 & -450 & 0.7 & 50.50 \\
\hline Se-Se-Ge & 570 & 240 & 290 & 0.85 & 10.35 \\
\hline
\end{tabular}




\section{Figure Captions}

\section{Figure 1}

Glass formation region in the AGGS system. Blue area denotes homogenous glass formation and squares indicate homogenous glasses synthesized for this study. The surrounding green area corresponds to glass formation where glasses are phase separated (circles). Red crosses mark the compositions that devitrify upon cooling with ice water.

\section{Figure 2}

Representative DSC traces for a homogeneous AGGS glass with $\mathrm{R}=1$ (red) and a heterogeneous AGGS glass with $\mathrm{R}=0.25$ (green), both containing $50 \mathrm{~mol} \% \mathrm{GeSe}_{2}$. The onset of the glass transition is taken as $\mathrm{T}_{\mathrm{g}}$ and its locations are denoted with the intersection of lines. The blue line corresponds to the DSC trace for a Ga-free phase separated glass of composition $\mathrm{Ag}_{1.5} \mathrm{Ge}_{3} \mathrm{Se}_{9}$ composition, showing two glass transitions.

\section{Figure 3}

Unpolarized Raman spectra of AGGS glasses for (a) the compositional series with 10 mol\% $\mathrm{Ga}_{2} \mathrm{Se}_{3}$ and variable $\mathrm{Ag}_{2} \mathrm{Se}$ concentration. The composition series with $\mathrm{R}=1$ as a function of the $\mathrm{GeSe}_{2}$ content (b) and the composition series with nearly constant (7.5 to $\left.10 \mathrm{~mol} \%\right) \mathrm{Ag}_{2} \mathrm{Se}$ content but variable $\mathrm{Ga}_{2} \mathrm{Se}_{3}: \mathrm{GeSe}_{2}$ ratio (c). The dashed line aids in showing the shift in the band intensity with increasing $\mathrm{Ag}_{2} \mathrm{Se}$ content in (a) and (c).

\section{Figure 4}

Experimental (black) and simulated (red) $11.7 \mathrm{~T}{ }^{77}$ Se MAS line shapes for AGGS glasses with $10 \mathrm{~mol} \% \mathrm{Ga}_{2} \mathrm{Se}_{3}$ and variable $\mathrm{Ag}_{2} \mathrm{Se}$ concentration. Simulation components corresponding to Se-Se-Ge, Ge-Se-Ge and Ge-Se-Ag sites are shown in green, blue and pink, respectively. The simulation parameters are listed in Table 2.

\section{Figure 5}

(a) Contour plot of the 2D ${ }^{77} \mathrm{Se}$ MATPASS/CPMG spectrum for the AGGS glass of composition $\left(\mathrm{Ag}_{2} \mathrm{Se}\right)_{40}\left(\mathrm{Ga}_{2} \mathrm{Se}_{3}\right)_{10}\left(\mathrm{GeSe}_{2}\right)_{60}$ with the total isotropic projection (top) and the two anisotropic projections (right) along the dotted lines A and B. (b) The experimental (black line) and simulated (red line) isotropic projection of the MATPASS/CPMG spectrum. Simulation components corresponding to Se-Se-Ge, Ge-Se-Ge and Ge-Se-Ag (NBSe) sites are shown in green, blue and pink, respectively. (c) The experimental (black solid lines) and simulated (red dotted lines) CSA sideband patterns taken at the isotropic chemical shifts of the Ge-Se-Ge and Ge-Se-Ag environments . 


\section{Figure 6}

Comparison between composition dependence of the fraction of NBSe sites obtained from simulated MAS line shapes in Fig. 4 (black squares) and the corresponding values (red circles) calculated from stoichiometry (see text for details). Data point denoted by the blue diamond is obtained from simulation of the MATPASS/CPMG isotropic spectrum in Fig. 5b.

\section{Figure 7}

(a) Experimental (black and purple) and simulated (green) ${ }^{71}$ Ga QMAT NMR spectra for two AGGS glasses with $60 \% \mathrm{GeSe}_{2}$ and $\mathrm{R} \leq 1$. The glass compositions are shown alongside the spectra.

b) Experimental (black) and simulated (green) ${ }^{71} \mathrm{Ga}$ (top) and ${ }^{69} \mathrm{Ga}$ (bottom) QMAT NMR spectra for AGGS glass with $60 \% \mathrm{GeSe}_{2}$ and $\mathrm{R}=4$. Both spectra are simulated with three components, each with a Czjzek model of distribution of $\mathrm{C}_{\mathrm{Q}}$. See text for details.

\section{Figure 8}

Compositional variation of $\mathrm{T}_{\mathrm{g}}$ in AGGS glasses (a) as a function $\mathrm{R}$ for different $\mathrm{Ga}_{2} \mathrm{Se}_{3}$ contents and (b) as a function of $\mathrm{GeSe}_{2}$ content for glasses with $\mathrm{R}<1$ (circles) and $\mathrm{R}=1$ (triangles). The error bars are within the size of the symbols.

\section{Figure 9}

Compositional variation of molar volume of AGGS glasses as a function of $\mathrm{GeSe}_{2}$ content for $\mathrm{R}<1$ (triangles); $\mathrm{R}=1$ (squares); $\mathrm{R}>1$ (circles). The molar volume for glassy $\mathrm{GeSe}_{2}$ (inverted triangle) from [5] is also shown for comparison. 


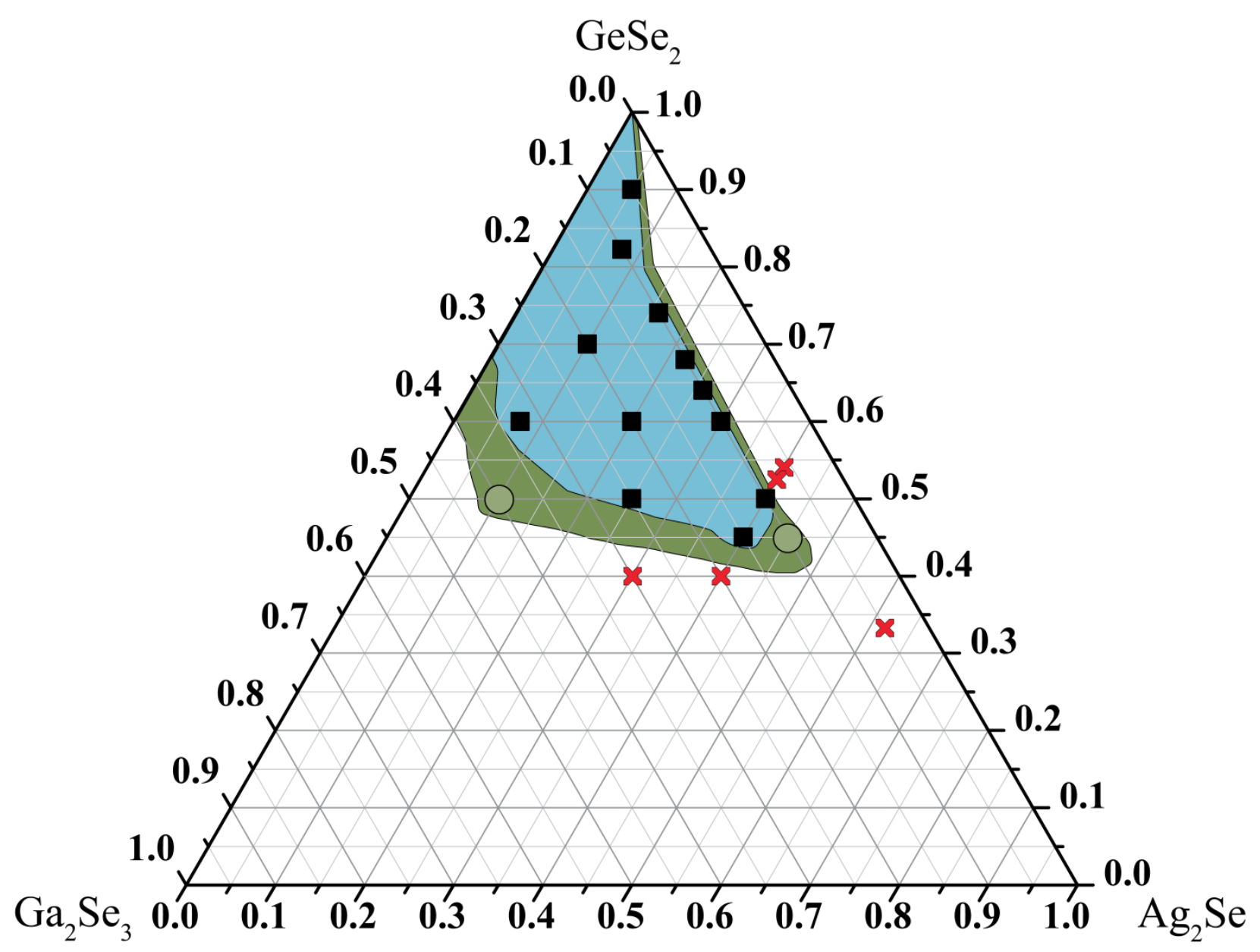

Fig. 1 


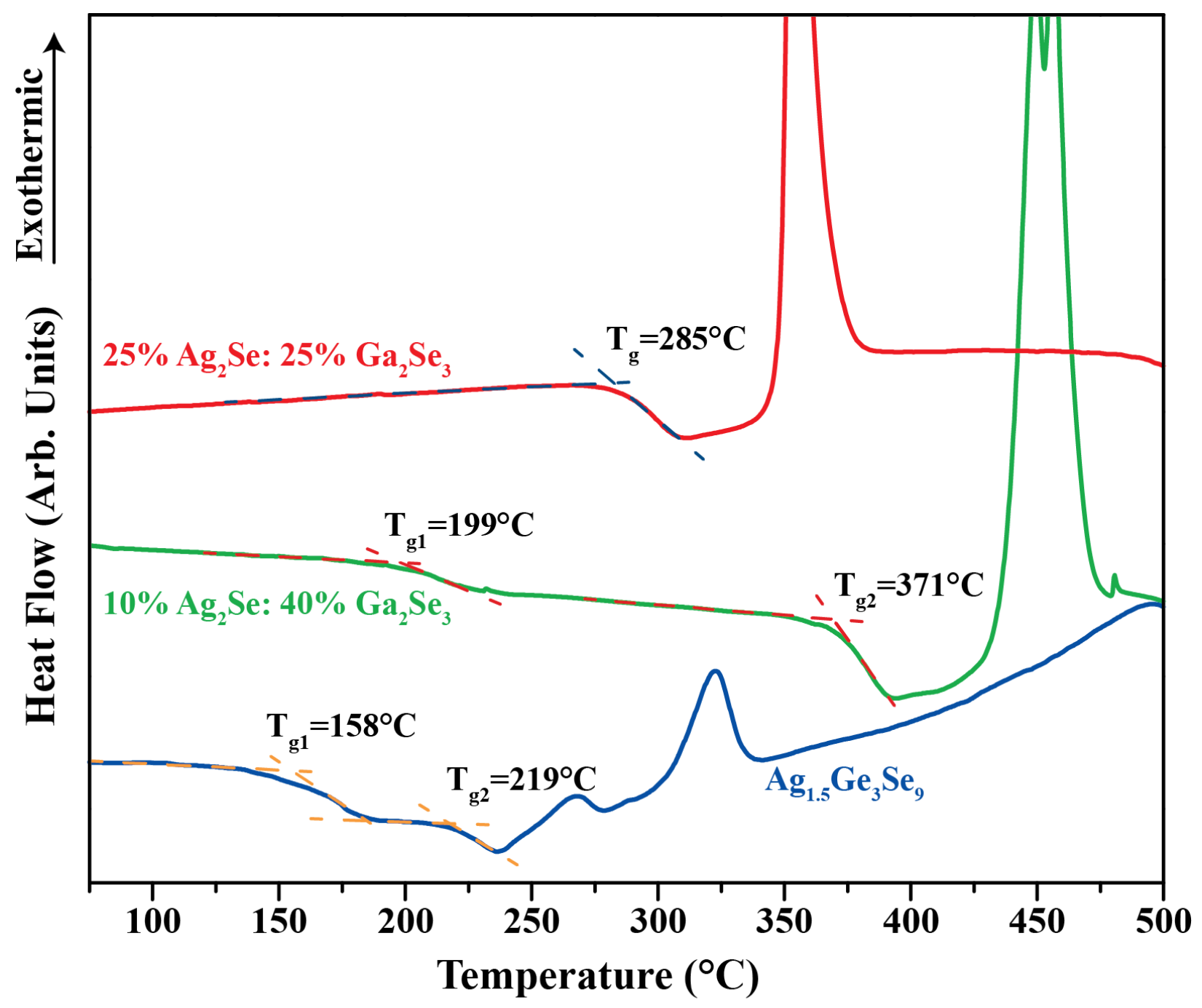

Fig. 2 


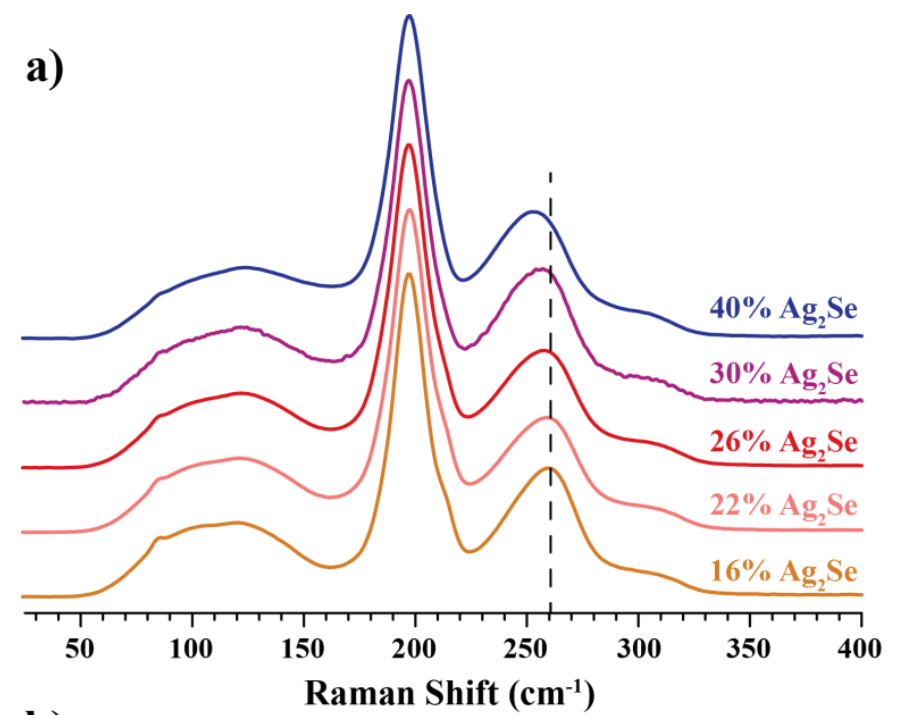

b)

Raman Shift $\left(\mathrm{cm}^{-1}\right)$

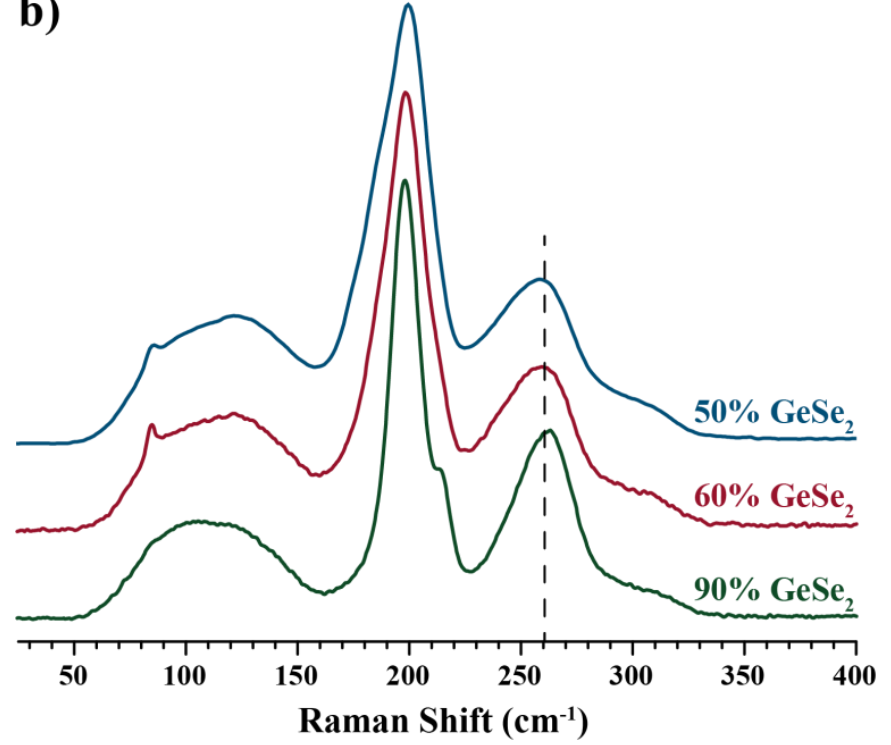

c)

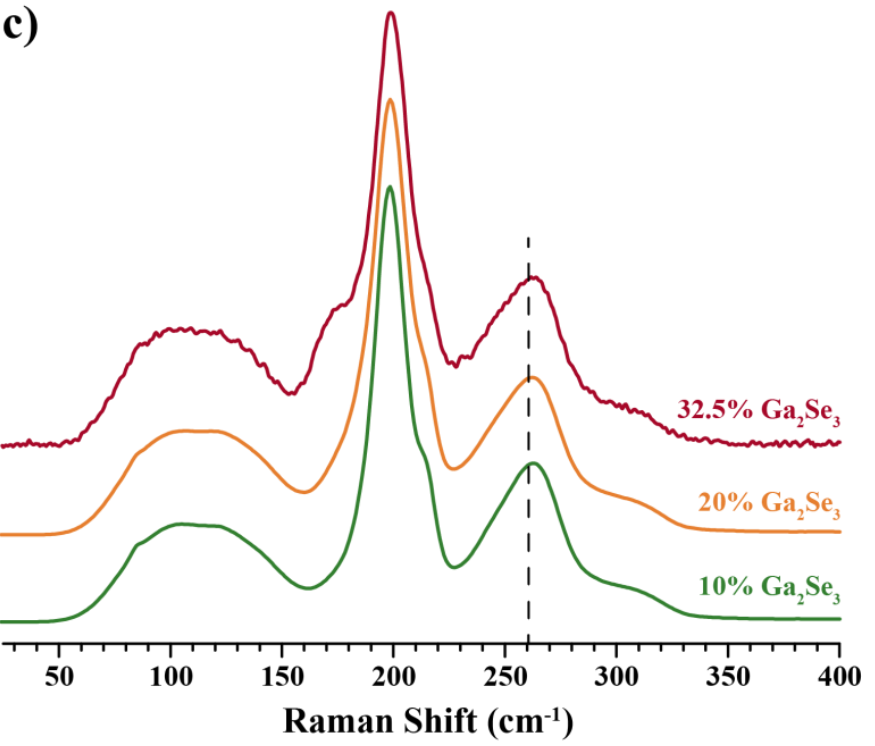

Fig. 3 

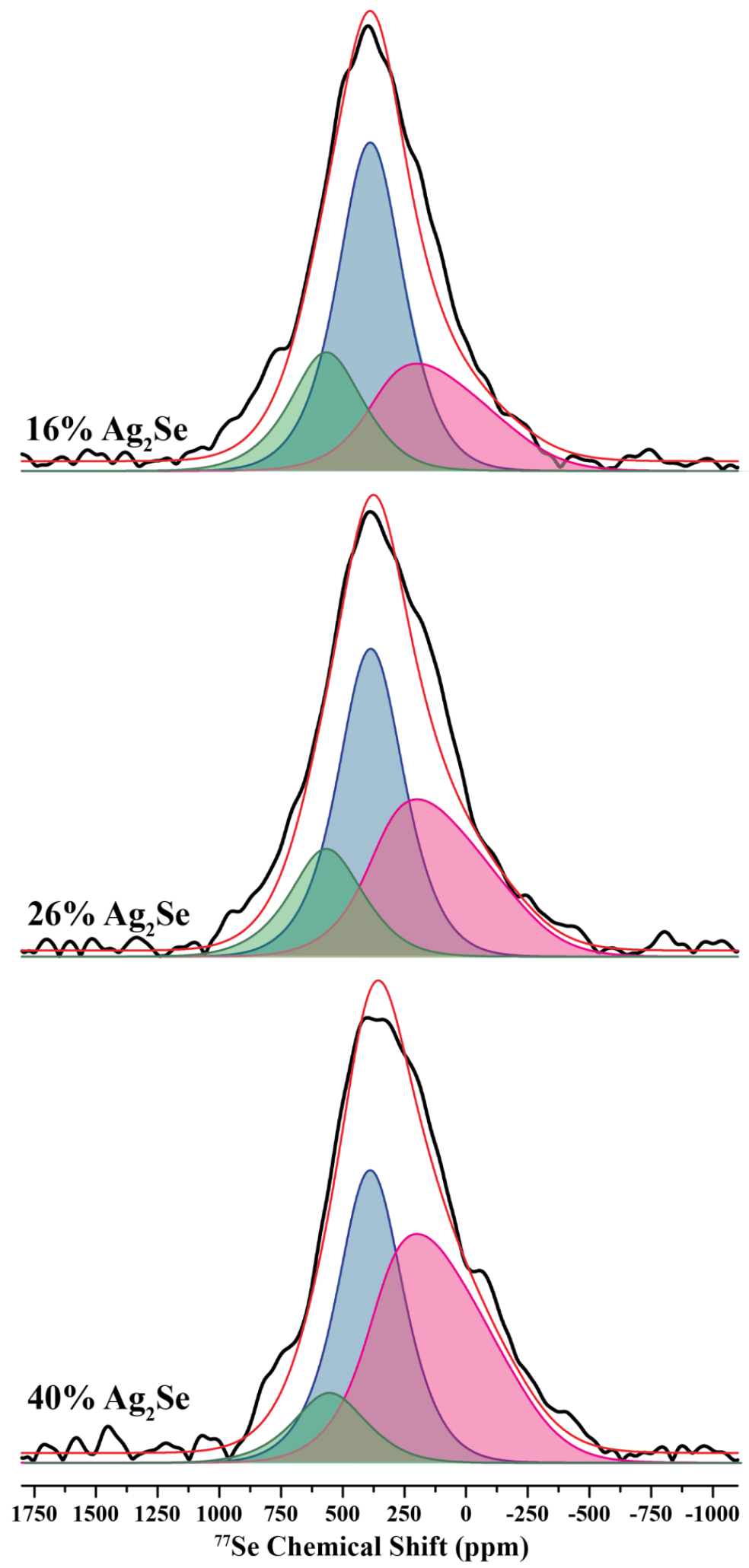

Fig. 4 


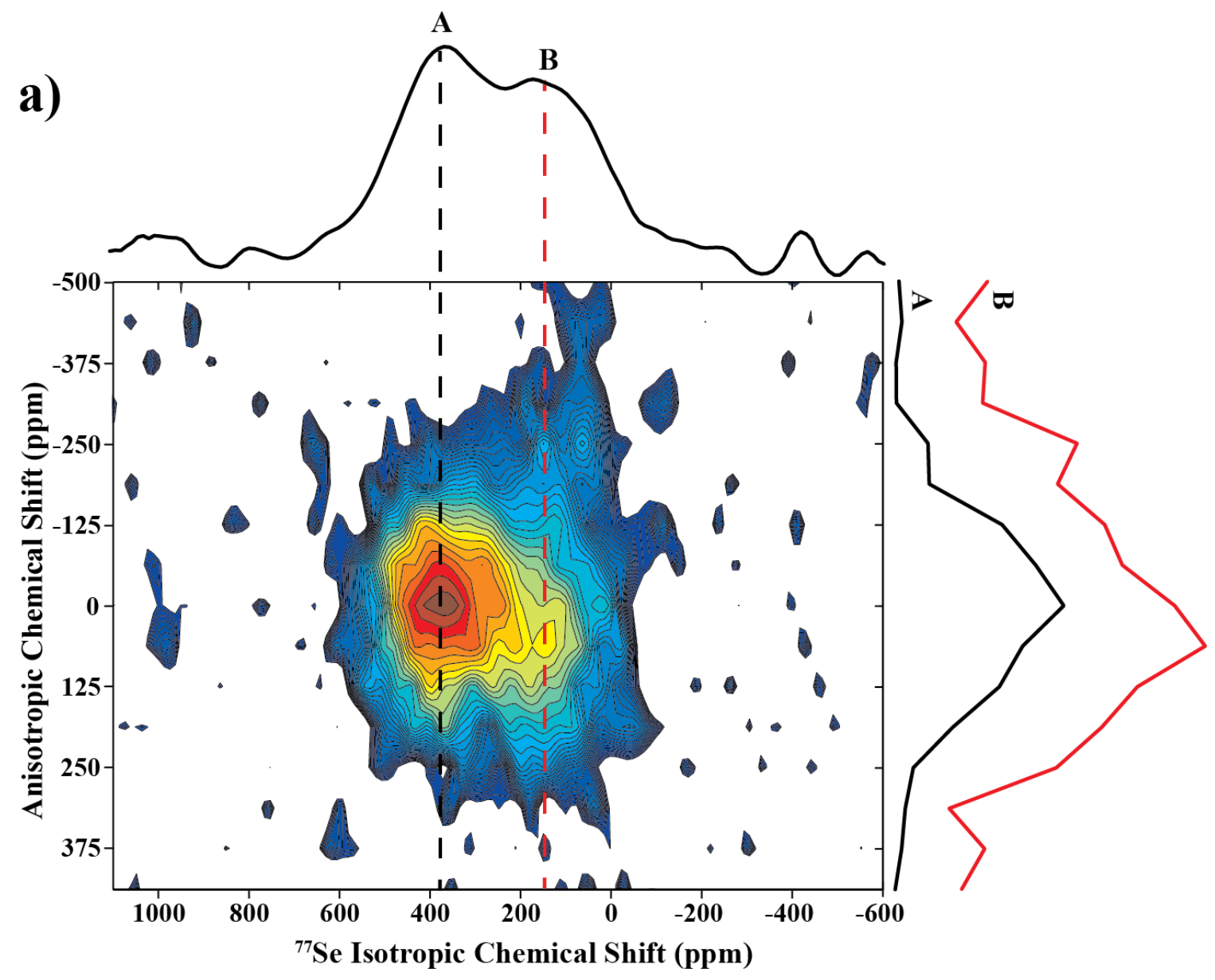

b)

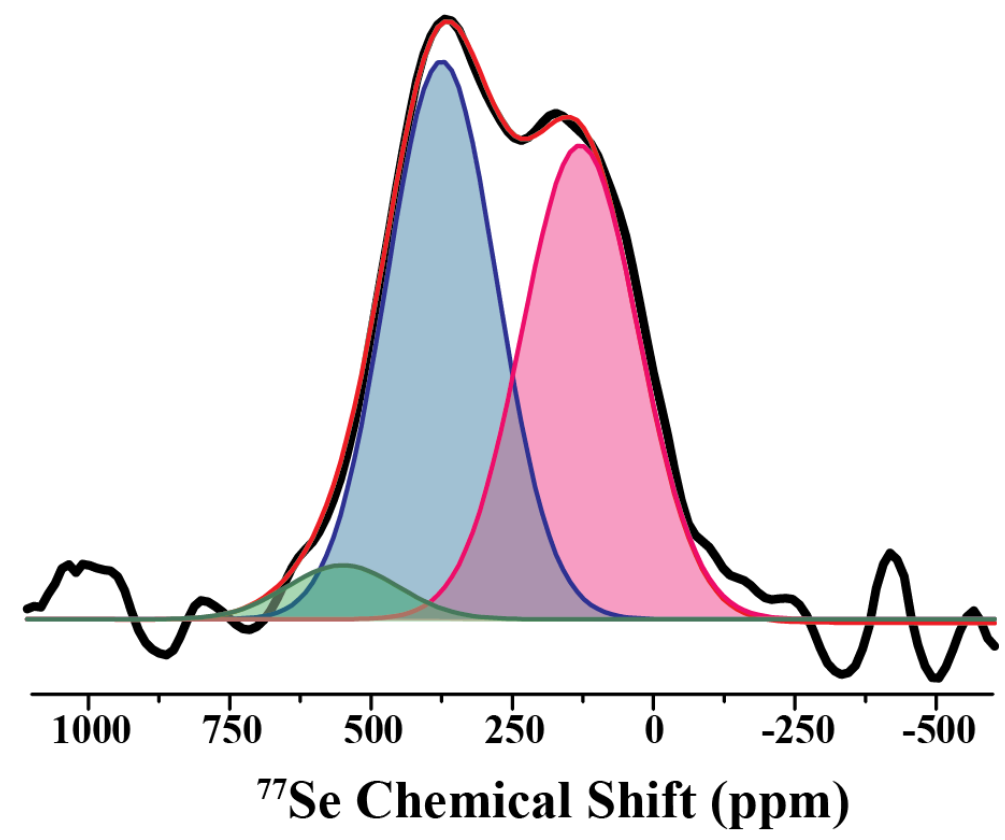

Fig. 5 
c)
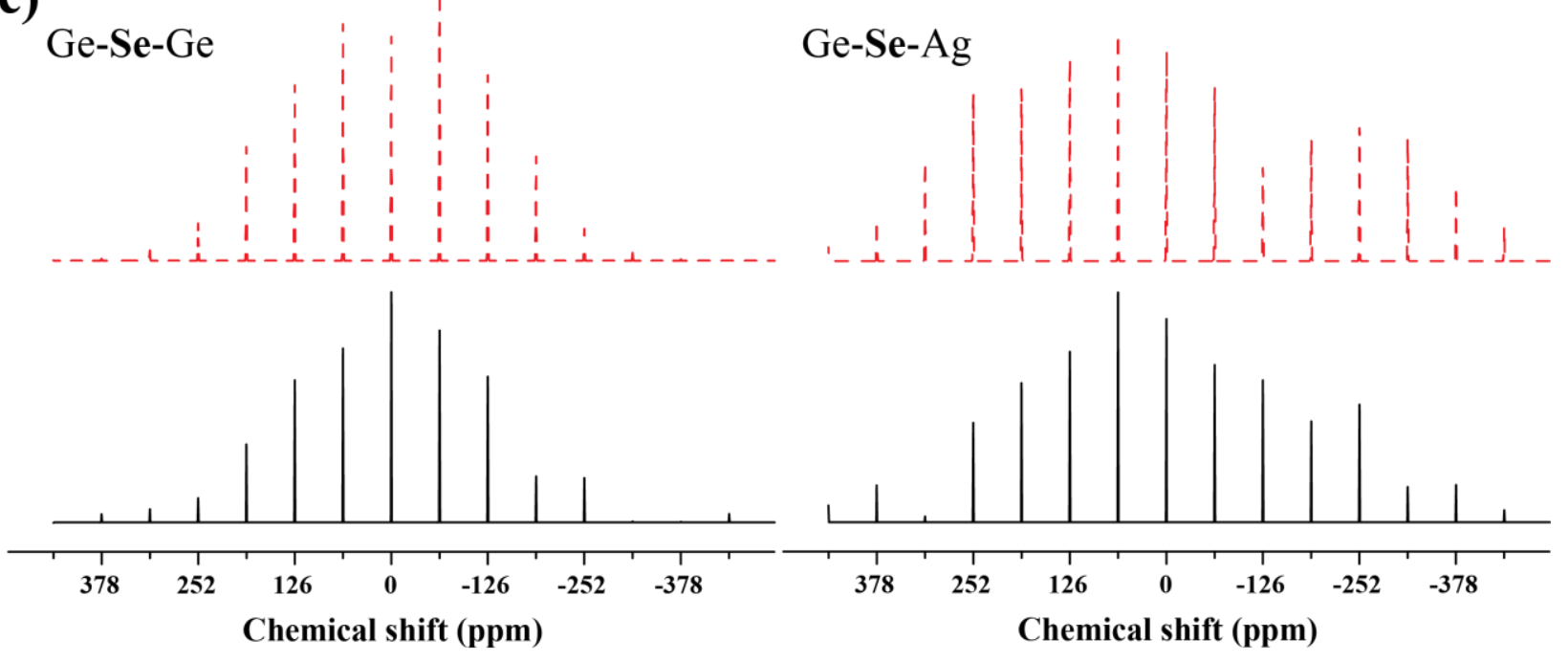

Fig. 5 


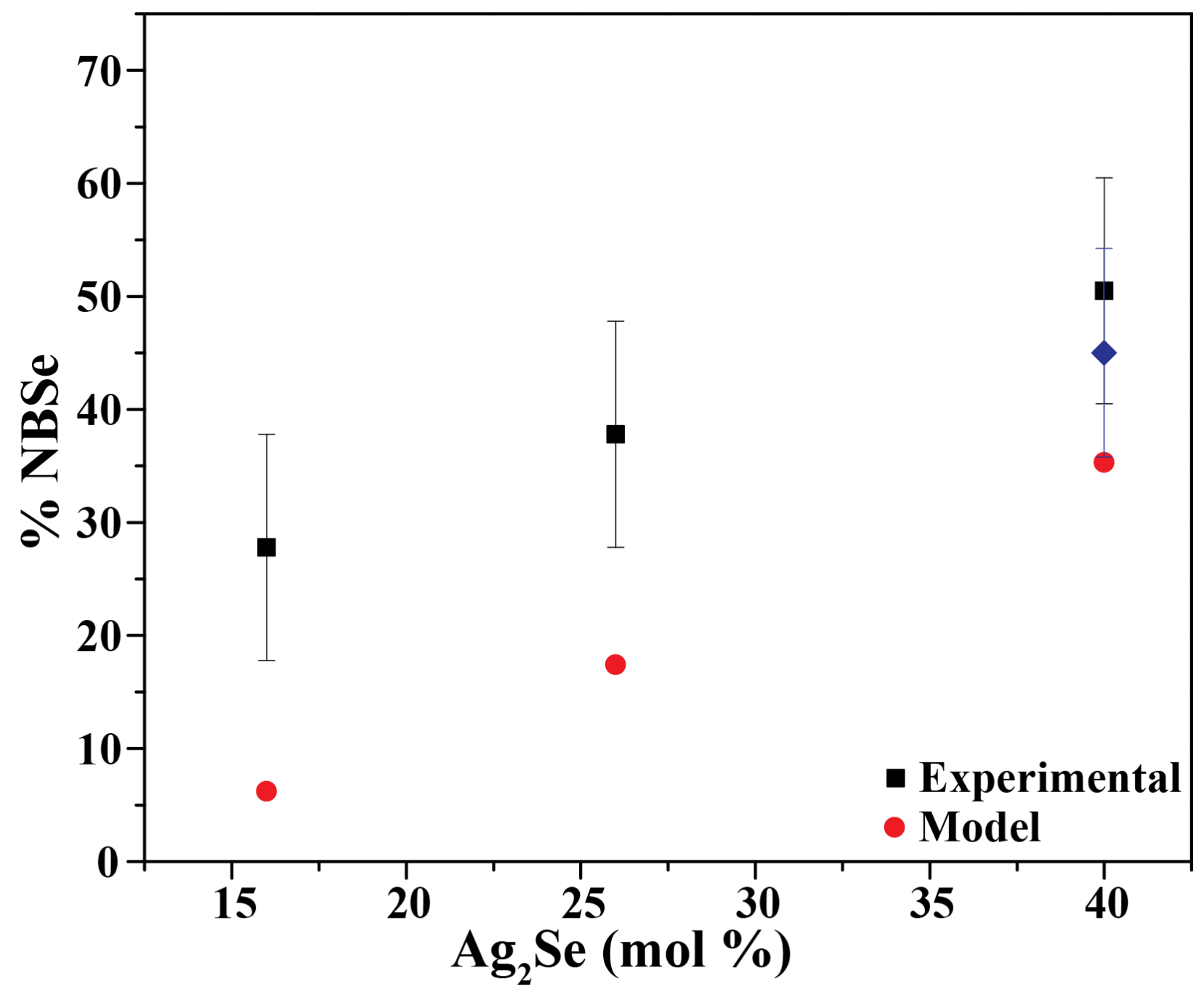

Fig. 6 


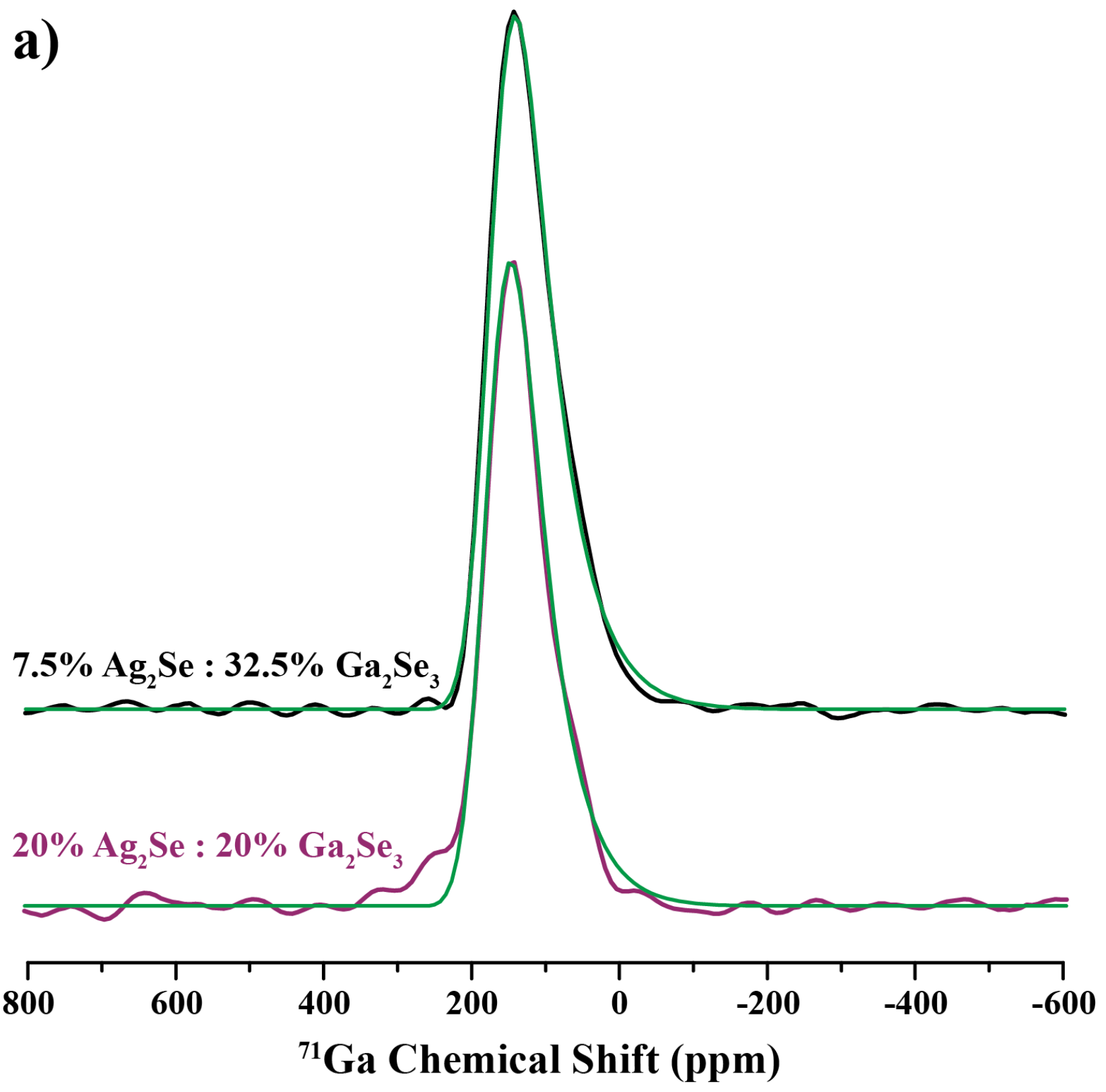

Fig. 7 


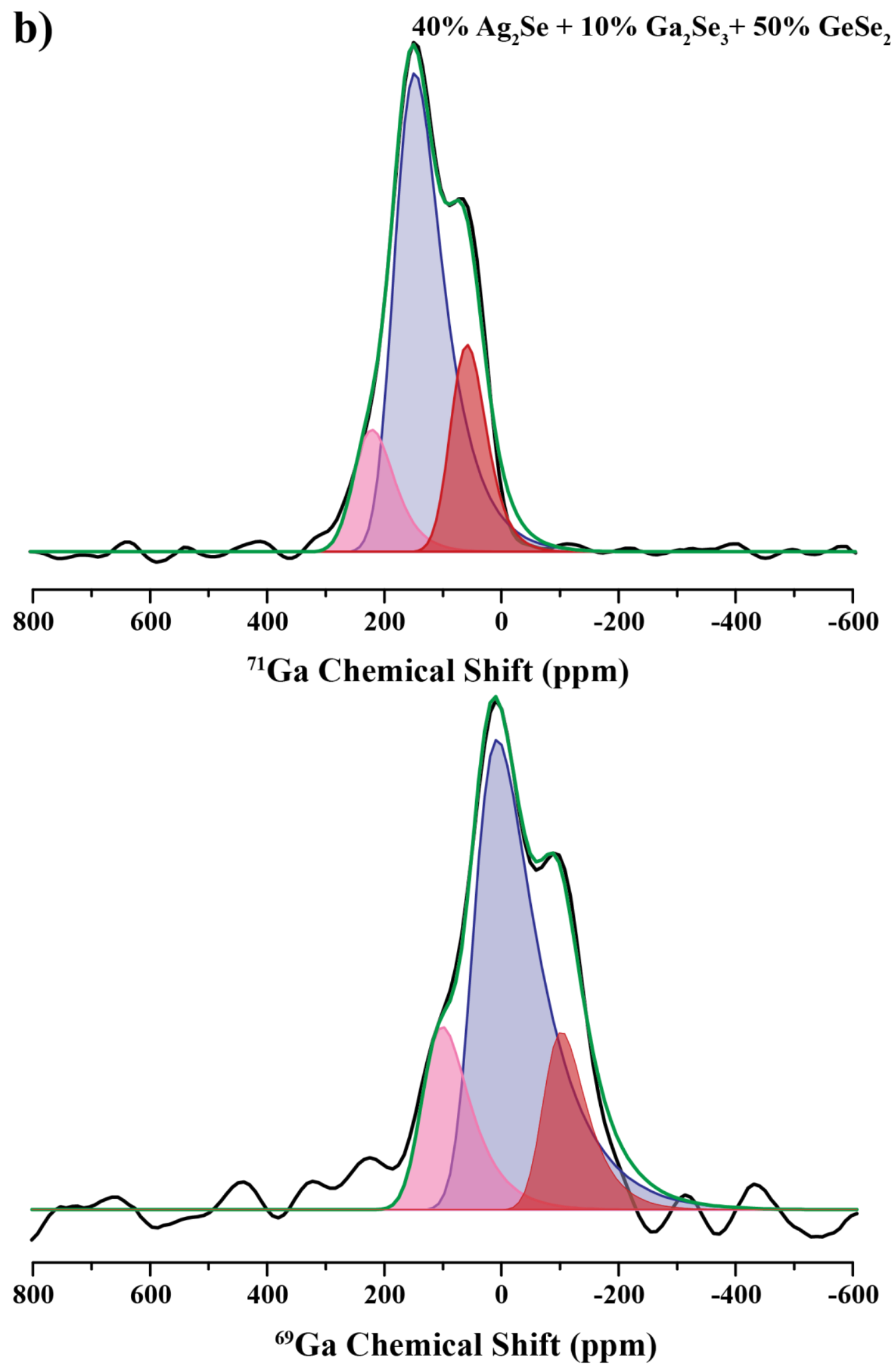

Fig. 7 
a)

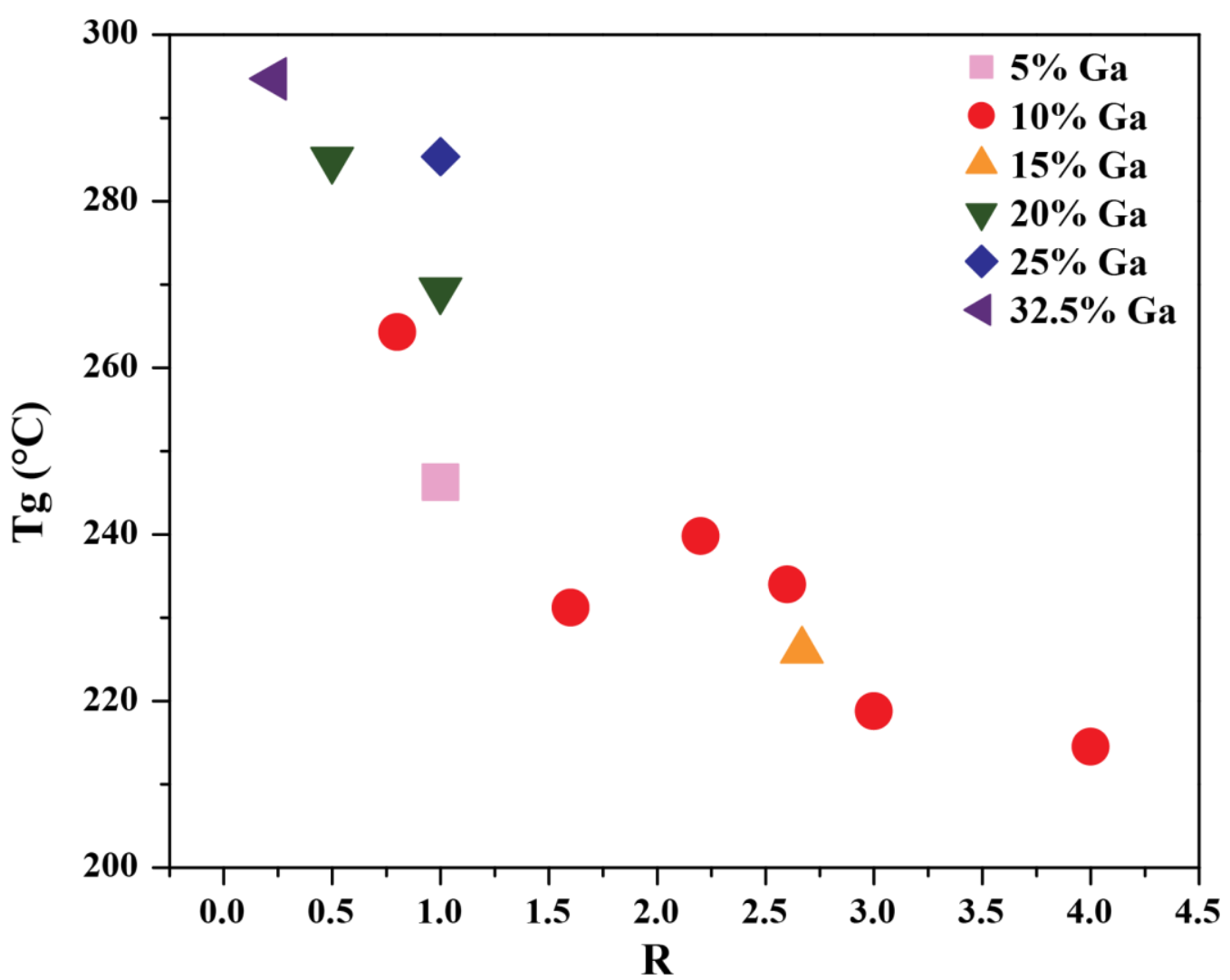

b)

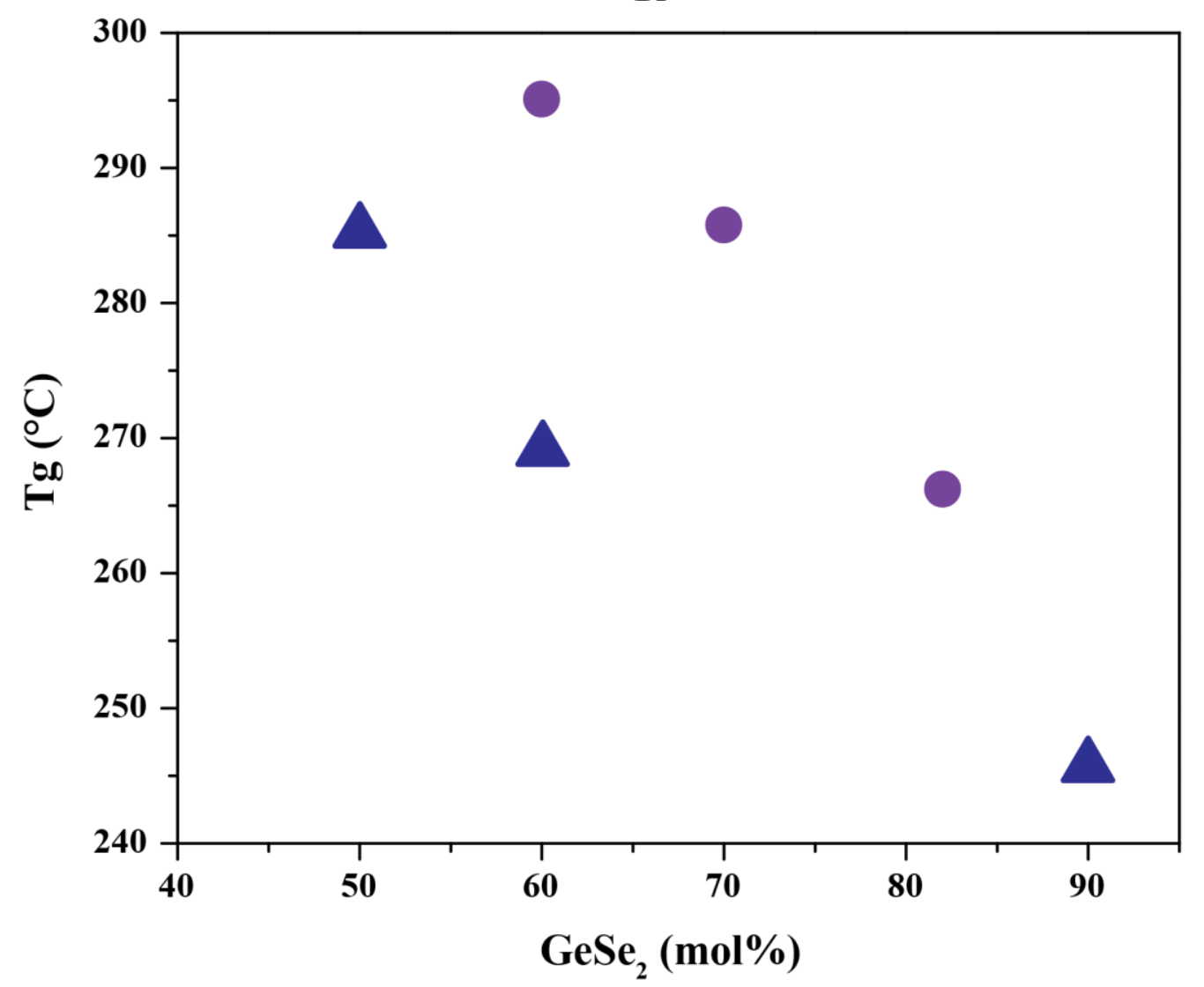

Fig. 8 


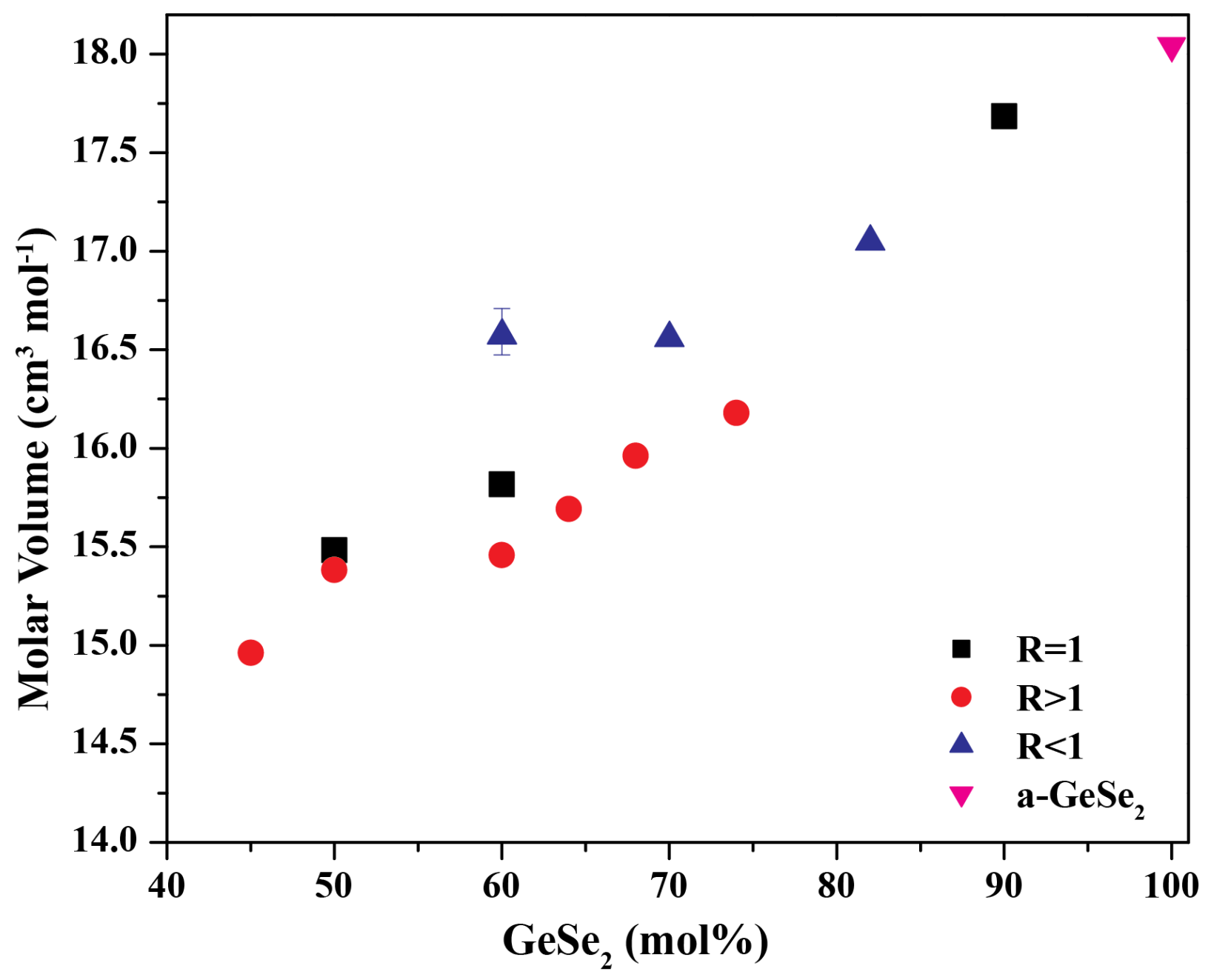

Fig. 9 